\title{
OPEN HLA-DRB1 allelic epitopes that associate with autoimmune disease risk or protection activate reciprocal macrophage polarization
}

\author{
Vincent van Drongelen ${ }^{1}$, Bruna Miglioranza Scavuzzi ${ }^{1}$, Sarah Veloso Nogueira ${ }^{1}$, \\ Frederick W. Miller ${ }^{2}$, Amr H. Sawalha ${ }^{1,3}$ \& Joseph Holoshitz ${ }^{1 凶}$
}

Associations between particular human leukocyte antigen (HLA) alleles and susceptibility to-or protection from-autoimmune diseases have been long observed. Allele-specific antigen presentation (AP) has been widely proposed as a culprit, but it is unclear whether HLA molecules might also have non-AP, disease-modulating effects. Here we demonstrate differential macrophage activation by $H L A-D R B 1$ alleles known to associate with autoimmune disease risk or protection with resultant polarization of pro-inflammatory ("M1") versus anti-inflammatory ("M2") macrophages, respectively. RNA-sequencing analyses of in vitro-polarized macrophages in the presence of AP-incompetent short synthetic peptides corresponding to the third allelic hypervariable regions coded by those two HLA-DRB1 alleles showed reciprocal activation of pro- versus anti-inflammatory transcriptomes, with implication of corresponding gene ontologies and upstream regulators. These results identify a previously unrecognized mechanism of differential immune modulation by short HLA-DRB1-coded allelic epitopes independent of AP, and could shed new light on the mechanistic basis of HLA-disease association.

It has been long observed that certain human leukocyte antigen (HLA) alleles confer higher risk for autoimmune diseases, while other alleles provide protective effects ${ }^{1}$. The molecular mechanisms underlying these associations are incompletely understood. Modeled on the major histocompatibility complex (MHC)-restricted antigen presentation (AP) paradigm ${ }^{2,3}$, it has been hypothesized that HLA-associated diseases are triggered by presentation of self-antigens-or foreign antigens that cross-react with self by HLA-coded molecules ${ }^{4-7}$. It is worth noting, however, that the identity of the target antigens remains unknown in many HLA-associated conditions.

Compelling evidence of antigen-specific immune response exists in several HLA-associated conditions, such as celiac disease (Reviewed in ${ }^{8}$ ), or type I diabetes mellitus (Reviewed in ${ }^{9}$ ). In many other diseases, however, a candidate target antigen has not been yet identified, or AP might not be the sole mechanistic basis for their HLA associations, as previously discussed ${ }^{10,11}$. Two emblematic HLA-associated diseases, ankylosing spondylitis (AS) and rheumatoid arthritis (RA) illustrate the quandary.

AS associates strongly with particular HLA-B27 subtypes ${ }^{12,13}$. While the search for arthritogenic peptides that these class I HLA antigens putatively present is underway, a recent study found no qualitative differences in peptide binding preferences among HLA-B27 subtypes that associate with AS, versus disease-unassociated subtypes ${ }^{14}$. Additionally, in a rat model of AS, class II-restricted CD4 ${ }^{+} \mathrm{T}$ cells, rather than class I-restricted CD8 ${ }^{+}$ $\mathrm{T}$ cells appear to be involved in disease pathogenesis (Reviewed in ${ }^{15}$ ). Moreover, it has been recently established that independent of their putative disease-enhancing AP properties, AS-associated HLA-B27 molecules have a unique propensity to activate endoplasmic reticulum stress and misfold intracellularly, thereby triggering overproduction of pro-arthritogenic cytokines ${ }^{15}$.

In RA, the majority of affected individuals carry HLA-DRB1 alleles that code for a susceptibility (or 'shared') epitope (SE) motif QKRAA, QRRAA, or RRRAA in position 70-74 of the DR $\beta$ chain ${ }^{16}$. However, the association is not disease-specific. SE-coding $H L A-D R B 1$ alleles have also been found to associate with type 1 diabetes, autoimmune hepatitis, polymyalgia rheumatica, temporal arteritis and Parkinson's disease ${ }^{17-21}$, among other

${ }^{1}$ Department of Internal Medicine, University of Michigan, Ann Arbor, MI 48109, USA. ${ }^{2}$ Environmental Autoimmunity Group, National Institute of Environmental Health Sciences, Research Triangle Park, Durham, NC 27709, USA. ${ }^{3}$ Present address: Departments of Pediatrics and Internal Medicine, University of Pittsburgh, Pittsburgh, PA 15224, USA. ${ }^{\varpi}$ email: jholo@med.umich.edu 
conditions. Further, the SE has been found to be a risk factor for erosive bone damage in nosologically-distinct conditions, such as periodontal disease, systemic lupus erythematosus and psoriatic arthritis ${ }^{22-26}$. Additionally, while AP by SE-expressing HLA-DR molecules has been long postulated to be a disease-susceptibility mechanism in RA, only minor overlaps were found among the repertoires of peptides eluted from different SE-expressing HLA-DR molecules (encoded by either $D R B 1^{\star} 01: 01$; $D R B 1^{\star} 04: 01$, or $D R B 1^{\star} 10: 01$ ). Notably, comparable overlaps were found with a non-SE-expressing $\left(D R B 1^{\star} 15: 01\right)$ molecule ${ }^{27}$.

An inverse disease association exists with $H L A-D R B 1$ alleles that encode a protective epitope (PE) motif 70-DERAA-74 (at the exact same region of the DR $\beta$ chain as the SE). Those alleles significantly reduce the risk for $\mathrm{RA}^{28-31}$, and many other autoimmune conditions, including systemic lupus erythematosus, mixed connective tissue disease, progressive systemic sclerosis, antineutrophil cytoplasmic antibodies (ANCA)-positive vasculitis, narcolepsy and myasthenia gravis ${ }^{32-37}$.

Thus, both the SE and PE modulate disease risk in a wide range of conditions that do not share a common putative target antigen, pathogenesis, or target tissues. To determine whether the SE and PE, aside from their AP function, may also possess AP-independent effects, here we compared their respective impacts on macrophage activation, given the pivotal role that these cells play in autoimmunity ${ }^{38-42}$. The findings demonstrate that primary bone marrow macrophages from naïve transgenic mice that constitutively express on their cell surface SE-positive HLA-DR molecules are polarized preferentially toward pro-inflammatory ("M1") macrophage, while macrophages from transgenic mice that express PE-positive HLA-DR molecules show enhanced antiinflammatory ("M2") polarization.

To exclude the possibility of AP of self- or tissue culture-derived antigens by the transgenic HLA-DR molecules, and to map the functional epitopes, we studied AP-incompetent soluble synthetic 15-mer peptides corresponding to the third allelic hypervariable regions (TAHRs) coded by these two HLA-DRB1 alleles. Using an RNA sequencing (RNA-seq) approach, we demonstrate here that consistent with the above findings, exposure of macrophages to SE- or PE-expressing peptides activates distinct transcriptomes. A SE-expressing peptide activates genes, biological processes and upstream regulators known to mediate pro-M1, pro-inflammation or pro-autoimmune disease effects, whereas a PE-expressing peptide facilitates activation of genes, biological processes and upstream regulators that are known to mediate pro-M2, anti-inflammatory, or anti-autoimmune disease effects. Thus, gene products coded by $H L A-D R B 1$ alleles that are known to associate with autoimmune disease susceptibility versus protection activate, respectively, pro-inflammatory or anti-inflammatory pathways in an AP-independent fashion.

\section{Results}

HLA-DRB1 allele-specific differential macrophage polarization in transgenic mice. To determine whether autoimmune disease risk or protective $H L A-D R B 1$ alleles have distinct effects on macrophage polarization we first studied ex vivo primary bone marrow-derived macrophages (BMDMs) isolated from transgenic mice ${ }^{43,44}$ that express human HLA-DR $\beta$ chains coded by the $D R B 1^{* 04: 02}$ allele with a PE (70-DERAA-74) sequence in the TAHR ("PE Tg"), versus BMDMs from mice of the same genetic background expressing a SE (70-QKRAA-74) sequence, encoded by the RA-susceptibility allele DRB1*04:01 ("SE Tg"). The TAHRs of the two transgenic mouse lines differ by only 3 amino acid residues. As shown in Fig. 1, under M1-polarizing culture conditions, SE Tg-derived BMDMs expressed higher levels of the M1 gene markers Cxcl10, Nos2, Il-12p40, Il-1b, Tnfa, Il-6 and Ccl 2 compared to PE Tg-derived BMDMs (Fig. 1A). Additionally, SE Tg-derived BMDMs produced Il-6, Tnfa, and Il-12p70 proteins at significantly higher levels than PE Tg-derived BMDMs (Fig. 1B). SE Tg BMDMs also showed higher baseline levels of nitric oxide (NO) production with a significant increase under M1 polarizing culture conditions, whereas PE Tg-derived BMDMs did not produce any NO in either culture conditions (Fig. 1C). A mirror-image pattern was found under M2-polarizing culture conditions: PE Tg BMDMs demonstrated higher expression of the M2 gene markers Arg1, Ym1 and Ccl17 relative to SE Tg BMDMs (Fig. 1D). Consistent with the above findings, and with the role of arginase in M2 polarization ${ }^{45}$, a significantly increased arginase activity under M2 polarizing conditions was found in BMDMs from PE Tg, compared to SE Tg (Fig. 1E).

HLA-DRB1 allele-based differential macrophage polarization was found in vivo as well. As shown in Fig. 2A, intra peritoneal (i.p.) administration of an M1-polarizing agent (LPS) significantly increased gene expression of M1 markers Il12p40, Il12p35, Il23p19, Il-1b, Tnfa, Ccl2 and Il-6 in peritoneal macrophages from SE Tg, compared to PE Tg mice. Additionally, SE Tg mice produced higher serum levels of various pro-inflammatory, M1-associated cytokines, including Il-12, TNFa, GM-CSF and IFN $\gamma$ (Fig. 2B).

Signal transduction pathways. To better understand the mechanisms involved in HLA-DRB1 allelebased macrophage polarization predilections, we sought to determine whether signaling events are involved. To this end we focused on the Akt axis, known to play a pivotal role in M1 versus M2 macrophage polarization ${ }^{46,47}$. As Fig. 3 shows, under M1 polarizing conditions, BMDMs from PE Tg mice displayed significantly increased Akt phosphorylation compared to BMDMs from SE Tg mice (Fig. 3A). Ly294002-mediated inhibition of Pi3K, an upstream regulator of Akt, allowed significant rescue of the expression of M1 gene markers $I l-1 b$ and $I l-$ 6 (Fig. 3B), as well as Il-6 and Tnfa cytokine levels (Fig. 3C). To better characterize the upstream signaling events that impact Akt activation, we measured phosphorylation of two Pi3K regulating phosphatases: PTEN and SHIP1 under M1 polarizing conditions. PTEN phosphorylation levels were not different between SE Tg and PE Tg BMDMs (Supplemental Fig. S1A). However, SHIP1, showed significantly lower phosphorylation levels in PE Tg BMDMs compared to SE Tg (Supplemental Fig. S1B).

Another important signaling mechanism in M1 polarization is NF- $\kappa B^{48}$, mapped downstream of $\mathrm{Akt}^{49}$. We therefore asked whether NF- $\mathrm{B}$ plays a role in $H L A-D R B 1$-associated macrophage polarization. Under M1 
A

Cxcl10
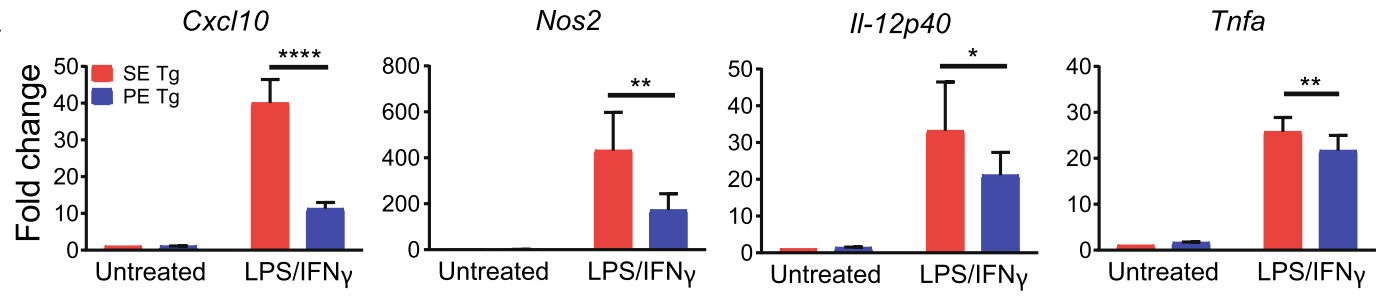

$I I-1 b$
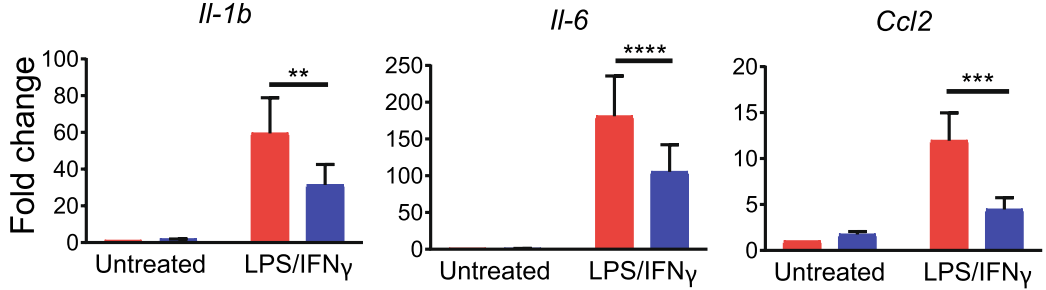

B
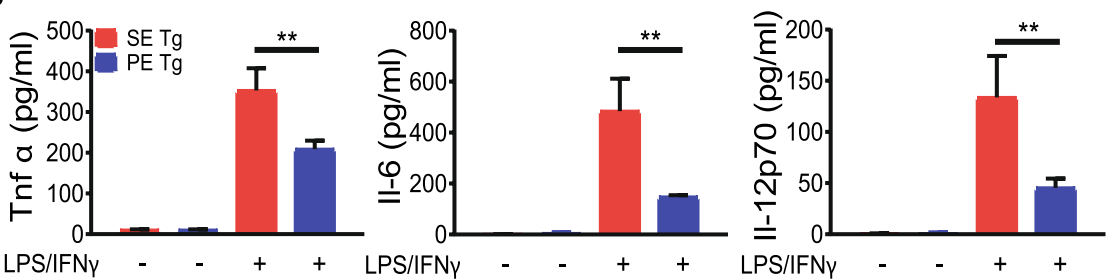

C

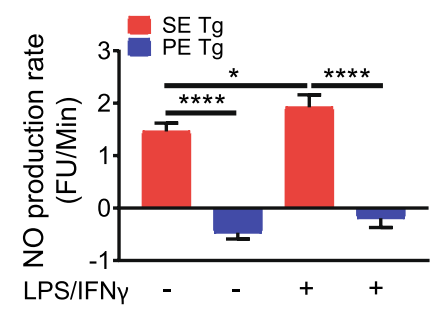

D

$\operatorname{Arg} 1$

$Y m 1$

$\mathrm{Ccl} 17$
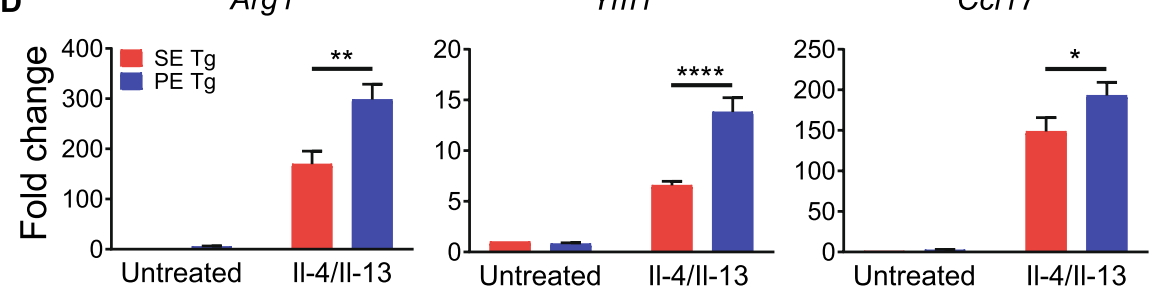

E

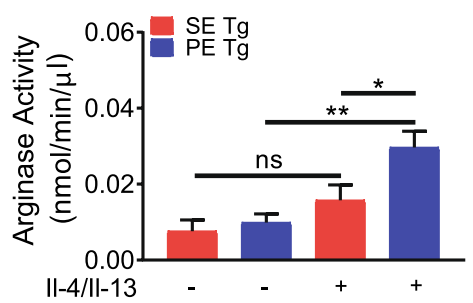

Figure 1. Differential in vitro macrophage polarization in SE Tg and PE Tg BMDMs. For M1 polarization, BMDMs were treated with LPS $(1 \mathrm{ng} / \mathrm{ml})+\operatorname{IFN} \gamma(20 \mathrm{ng} / \mathrm{ml})$ for $24 \mathrm{~h}$. M2 polarization was induced with Il-4 $(10 \mathrm{ng} / \mathrm{ml})+\mathrm{Il}-13(10 \mathrm{ng} / \mathrm{ml})$ for $24 \mathrm{~h}$. (A) qPCR for M1-associated genes. Data represent mean + SEM of 3-5 independent experiments. (B) ELISA for M1-associated cytokines. Data represent mean + SEM of 3 independent experiments. (C) NO production by BMDMs under M1 polarization conditions. Data represent mean + SEM of 8 replicates from 2 independent experiments. (D) qPCR for M2-associated genes. Data represent mean + SEM of 3-4 independent experiments. (E) Arginase activity in BMDMs under M2 polarization conditions. Data represent mean + SEM of 3 independent experiments. 2 -way ANOVA, ${ }^{\star} \mathrm{P}<0.05,{ }^{* *} \mathrm{P}<0.01,{ }^{* *} \mathrm{P}<0.001$, $* * * \star \mathrm{P}<0.0001$.

polarizing conditions, inhibition of NF- $\kappa \mathrm{B}$ decreased expression of M1 gene markers $\mathrm{Il}-1 \mathrm{~b}$, Nos 2 and $\mathrm{Ccl}$, as well as the M1 cytokine Il-12p70 in SE Tg BMDMs, but not in PE Tg BMDMs (Fig. 3D,E). Thus, taken together, we propose that the diminished M1 polarizability of PE Tg BMDMs is secondary to increased Akt activation, previously shown to inhibit NF- $\kappa B$ signaling ${ }^{46}$. We further propose that increased SHIP1 activity in SE Tg $\mathrm{BMDMs}$ results in reduced Akt activation, leading to enhanced NF- $\kappa \mathrm{B}$ activity, which, in turn leads to increased M1 polarization, consistent with previous studies ${ }^{46}$. A proposed model of the signaling pathways involved in $H L A-D R B 1$ allele-specific M1 macrophage polarization is shown in Fig. 3F.

Under M2 polarization conditions, significantly higher Akt phosphorylation was found in PE Tg BMDMs compared to SE Tg BMDMs (Fig. 4A). Modulation of the Akt signaling pathway through inhibition of Pi3K (upstream of Akt), or p70S6K (downstream of Akt), significantly suppressed expression of the M2 gene marker Arg1 (Fig. 4B,C, respectively). Noteworthy, another M2 gene marker Ym1 was not affected by either Pi3K or p70S6K inhibitors, suggesting that, different from Arg1, increased Ym1 gene expression in PE Tg BMDMs is Akt-independent. The possibility that $Y m 1$ gene expression in PE Tg BMDMs is controlled by Stat6, as previously reported in other cell ${ }^{50}$, is consistent with significantly higher Stat6 phosphorylation levels under M2 polarization conditions in PE Tg, compared to SE Tg BMDMs (Supplemental Fig. S2). A proposed model of the signaling pathways involved in $H L A-D R B 1$ allele-specific M2 macrophage polarization is shown in Fig. 4D.

HLA-DRB1 allele-specific transcriptome activation. To more conclusively determine whether the differential effects of the two HLA-DRB1 alleles may be AP-independent and to determine whether their effects could be mapped to the TAHR, we used AP-incompetent 15-mer synthetic peptides corresponding to the TAHR of the DR $\beta$ chain. 
A
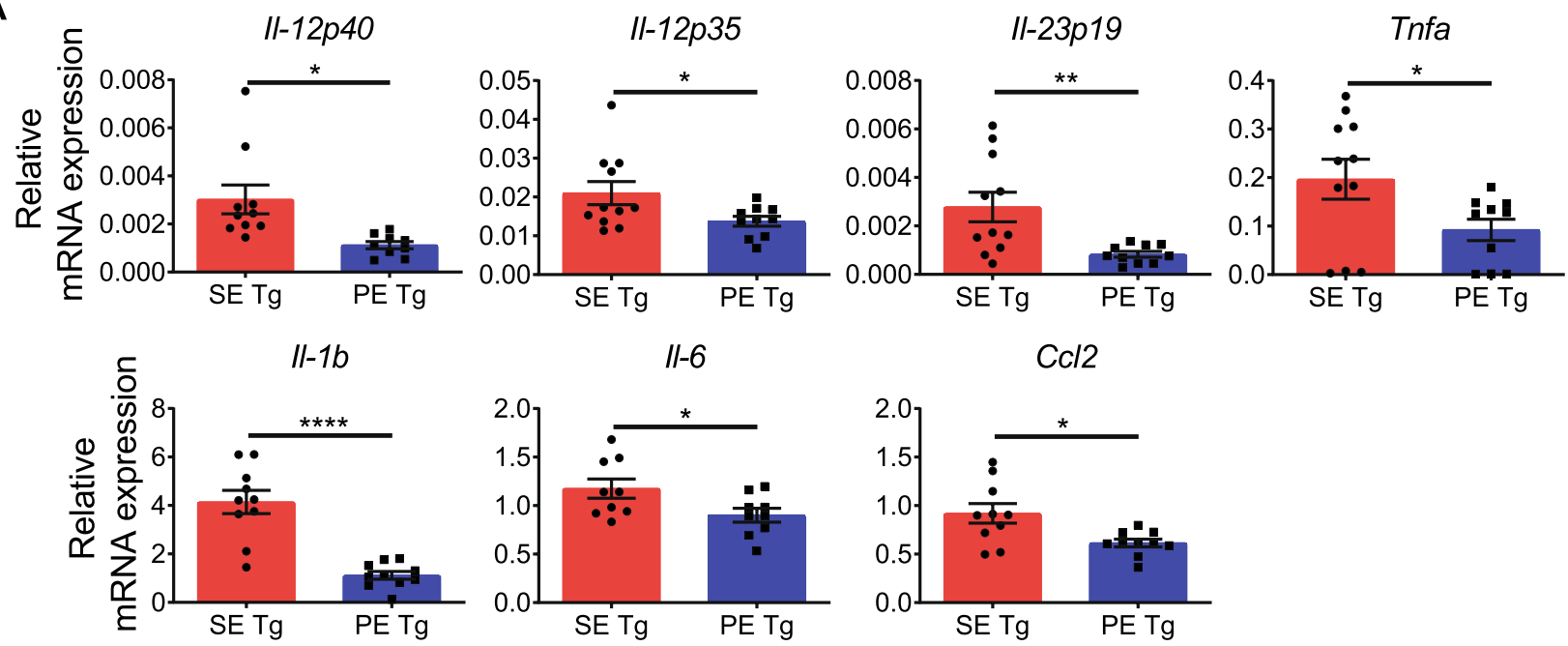

B
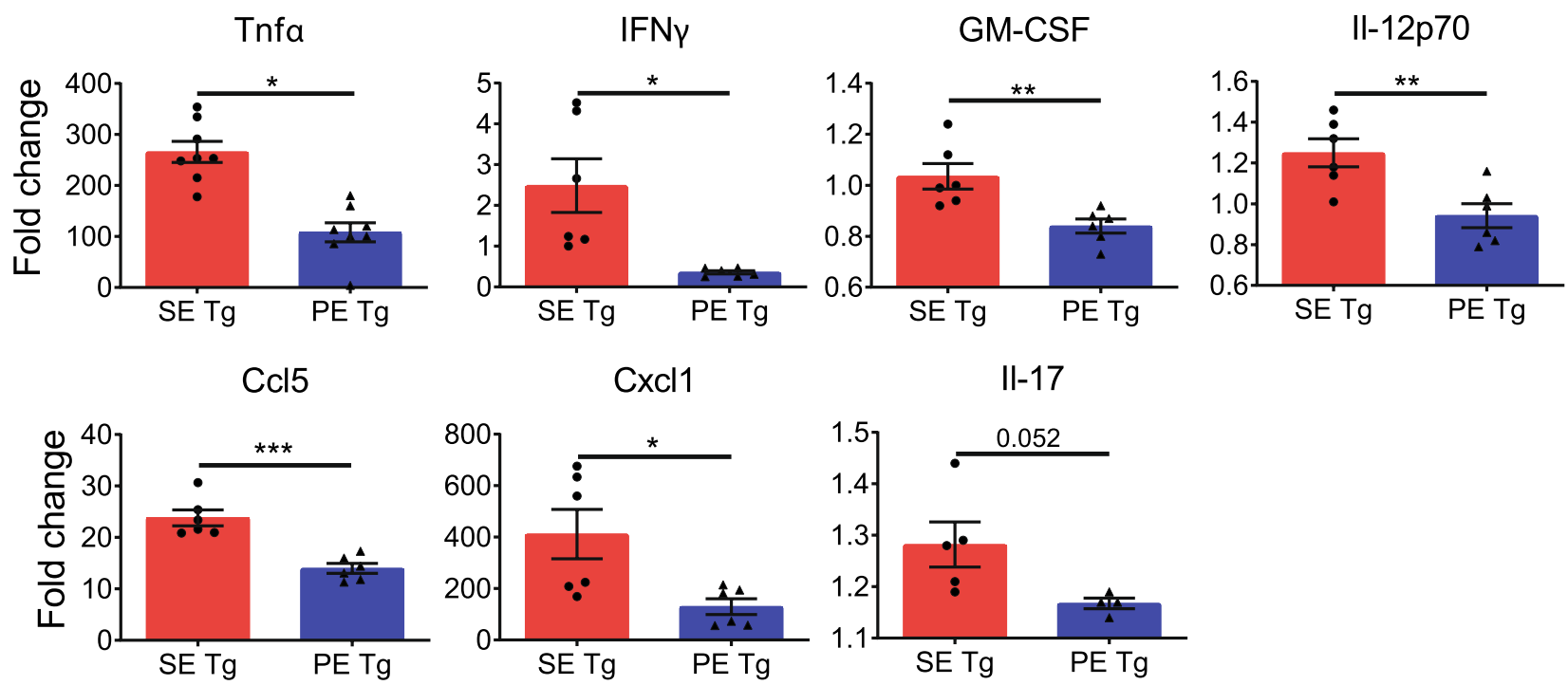

Figure 2. Differential in vivo macrophage polarization in SE Tg and PE Tg mice. To induce M1 polarization in vivo, mice were injected i.p. with LPS $(500 \mu \mathrm{g} / \mathrm{kg})$. Peritoneal macrophages and serum were collected after 4 h. (A) qPCR for mRNA expression of M1 marker genes in peritoneal macrophages. $n=8-11$. (B) ELISA for serum cytokine levels. $\mathrm{n}=5-8$. Results are compiled data from 3 experiments. Mean $\pm \mathrm{SEM}$, unpaired $t$ test with welch correction, ${ }^{\star} \mathrm{P}<0.05,{ }^{* *} \mathrm{P}<0.01,{ }^{* * *} \mathrm{P}<0.001,{ }^{* * *} \mathrm{P}<0.0001$.

To explore the feasibility of this approach, we first quantified expression levels of macrophage polarization marker genes in RAW 264.7 mouse macrophages following exposure to 15 -mer peptides designated "65-79*SE" or "65-79*PE", corresponding to amino acid residues 65-79 (TAHR) coded, respectively, by susceptibility (HLA$D R B 1^{\star} 04: 01$ ) or protective (e.g. $H L A-D R B 1^{\star} 04: 02, D R B 1^{\star} 13: 01$, or $D R B 1^{\star} 13: 02$ ) alleles. The 65-79^SE and $65-79^{*} \mathrm{PE}$ peptides differ by 3 amino acid residues, including only 2 substitutions in the $70-74$ region (QKRAA versus DERAA).

The rationale for studying these 15-mer peptides is based on previous studies, which demonstrated that $65-79^{*} \mathrm{SE}$ and its core 5-mer motif QKRAA activate pro-arthritogenic signal transduction and cell activation event ${ }^{51-55}$. These TAHR peptides were also found to carry out important arthritis-modulating functions. For example, soluble 65-79*SE and peptidomimetics of the QKRAA motif have been found to be arthritogenic in mice ${ }^{51,56}$, whereas $65-79^{*} \mathrm{PE}$ has been shown to dominantly protect against arthritis in mice carrying a permissive haplotype ${ }^{57}$, consistent with the findings that allele $H L A-D R B 1^{*} 04: 02$ is dominantly protective against arthritis in both mice ${ }^{44}$ and humans ${ }^{58}$. Additionally, Q70D substitution has a significant arthritis-protective effect in mice ${ }^{59}$, consistent with the role of D70 as a key PE residue for RA protection ${ }^{60}$.

As shown in Supplemental Figure S3, quantitative RT-PCR analysis confirmed that under M1-polarizing conditions, 65-79*SE, but not the 65-79*PE, activated transcription of the M1-marker genes Nos1, Cxcl10, Ccl2 and $\mathrm{Il}-1 \mathrm{~b}$ (Supplemental Fig. S3A). Under M2 polarizing conditions, 65-79* PE selectively upregulated expression of the M2 marker gene Mgl2 (Supplemental Fig. S3B). Thus, consistent with the findings in transgenic mouse BMDMs, 15 -mer peptides $65-79^{*} \mathrm{SE}$ and $65-79^{*} \mathrm{PE}$, corresponding to the TAHRs coded by DRB1 alleles that 
A
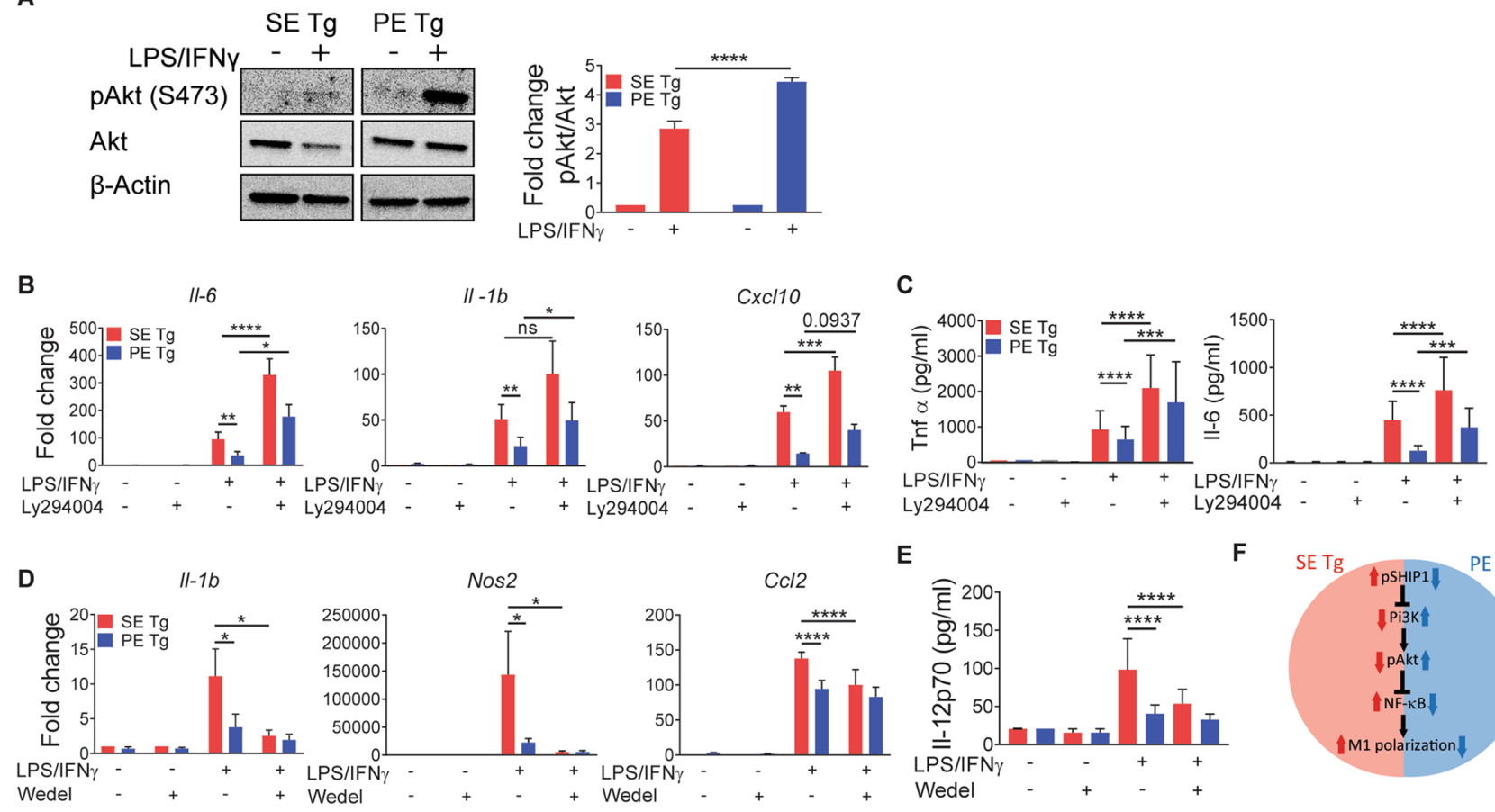

E
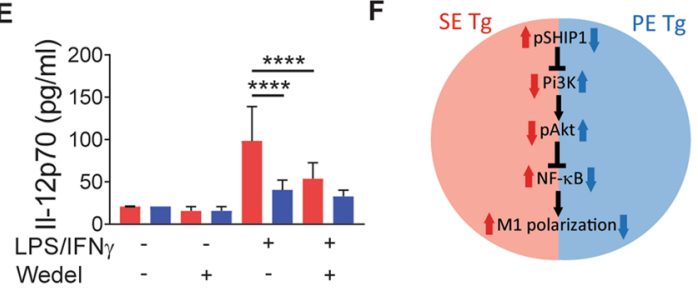

Figure 3. Involvement of signaling pathways in SE Tg and PE Tg BMDMs under M1 polarization conditions. M1 polarization in BMDMs was performed using the conditions described in Fig. 1. (A) Immunoblot for pAKt (Ser473) and Akt $15 \mathrm{~min}$. after exposure of cells to M1 polarization conditions. Quantification data represent mean + SEM of 3 independent experiments. (B-E) BMDMs were pre-treated with Ly294002 (5 $\mu$ M) $(\mathbf{B}, \mathbf{C})$, or wedelolactone $(10 \mu \mathrm{M})(\mathbf{D}, \mathbf{E})$ for $1 \mathrm{~h}$., followed by incubation in $\mathrm{M} 1$ polarization conditions as in $(\mathbf{A})$. (B,D) qPCR-based determination of M1 gene marker expression levels. (C and E) ELISA for Tnfa or Il-6 (C), or IL12p70 (E). (F) A proposed model of signaling pathway involvement under M1 polarizing conditions. Data represent mean and SEM of 3-5 independent experiments. Statistics: within group comparisons, paired $t$ test; between groups comparison, 2-way ANOVA, ${ }^{*} \mathrm{P}<0.05,{ }^{* *} \mathrm{P}<0.01,{ }^{* * *} \mathrm{P}<0.001,{ }^{* * *} \mathrm{P}<0.0001$.

confer autoimmune disease risk or protection, respectively, recapitulated the differentially induced expression of M1 versus M2 macrophage polarization gene markers in an epitope-specific fashion.

We next used an RNA-seq approach to characterize the broader transcriptional effects of the two TAHRs in mouse RAW 264.7 macrophages stimulated with $65-79^{\star}$ SE or $65-79^{\star} \mathrm{PE}$. Under M1 polarizing conditions (Fig. 5) the two TAHR peptides had a distinct effect on the number of upregulated and downregulated differentially expressed genes (DEGs) (Fig. 5A, Data File S1A). Among the unique upregulated genes induced by $65-79^{*}$ SE in M1-polarizing conditions (Fig. 5B,C, Supplemental Table S1, Data File S2A) were many RA disease marker-or risk factor-genes (e.g. Stat3, Cd44, Traf1, Tnfaip3), genes that encode confirmed or proposed therapeutic targets (e.g. Jak1, Jak3, Stat3, Ccr1, Cxcr4, Mmp14), and genes involved in autoimmune disease pathogenic mechanisms, such as NF- $\kappa$ B activation (e.g. Relb, Kpna4, Vav1), angiogenesis (Dusp4, Pgf, Egr1, Vegfc, Vegfa, Hbegf, Hmga1), Th17 polarization (e.g. Dusp4, Runx1, Hbegf), osteoclastogenesis (e.g. Atf4, Adam8, Dcstamp, Cxcr1), or M1 polarization (Klf6). Conversely, 65-79SE downregulated many anti-inflammatory (e.g. Akap1, Casp9, Cyp51,

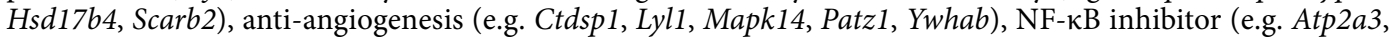
Bag2), and pro-M2 (e.g. Fads1, Klf2, Lpcat3, Pon2, Ywhab) genes. Representative modulated genes are shown in Fig. 5C. A list of notable genes, along with annotations and statistical significances, is shown in Supplemental Table S1. A complete list of unique 65-79*SE-modulated DEGs is shown in Data File S2A.

A diametrically opposite gene transcription pattern under M1-polarizing conditions was found with 65-79*PE (Fig. 5B, Data File S2B). It showed uniquely upregulated anti-inflammatory (e.g. Mt2, Il17rc, Havcr2, Ogg1, Nrf1, Stk10, Pdcd2), anti-angiogenesis (e.g. Flip1l, Fyn, Htatip2), anti-bone resorption (e.g. Def6, Gpr65, Bglap, Fbxl12), anti-oxidative (e.g. Mt2, Pycr1, Stc2, Dock3), and pro-M2 or anti-M1 (e.g. Tlr1, Nrf1, Themis2) genes. Conversely, unique downregulated genes by 65-79^PE included many pro-osteoclastogenic (e.g. Atp6v0d2, Dnmt3a, Syk, Ckb, Tspan5, Acp5, Src), pro-angiogenesis (e.g. St3gal1, Glul, Epn2, F7, Arhgap24), pro-arthritogenic (e.g. Syk, Jun, Tnfrsf9, F10, Arhgap24, Adamts7), and NF-kB pathway-activating (e.g. Sh3kbp1, Ddx58, F7) genes. Representative genes are shown in Fig. 5C. A list of notable genes, along with annotations and statistical significances, is shown in Supplemental Table S1. A complete list of unique $65-79 *$ PE-modulated DEGs is shown in Data File S2B.

Gene ontology (GO) analysis (Fig. 5D-F) revealed many GO processes that involve cytokine/chemokine signaling, innate immune response and positive regulation of NF- $\kappa B$ activity in macrophages stimulated with $65-79^{\star}$ SE under M1 polarizing conditions (Fig. 5E, Data File S1C). By contrast, GO terms for genes upregulated by $65-79 * \mathrm{PE}$ in $\mathrm{M} 1$ polarizing conditions included processes involving inhibitory effects on signal transduction, 
A

SE Tg PE Tg
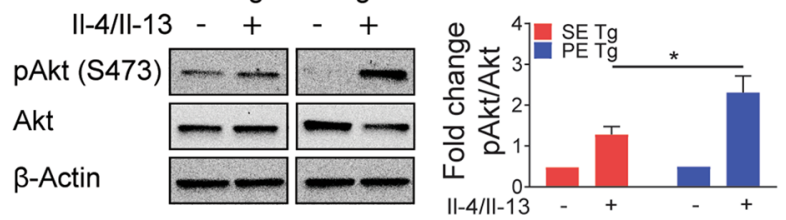

B

$\operatorname{Arg} 1$
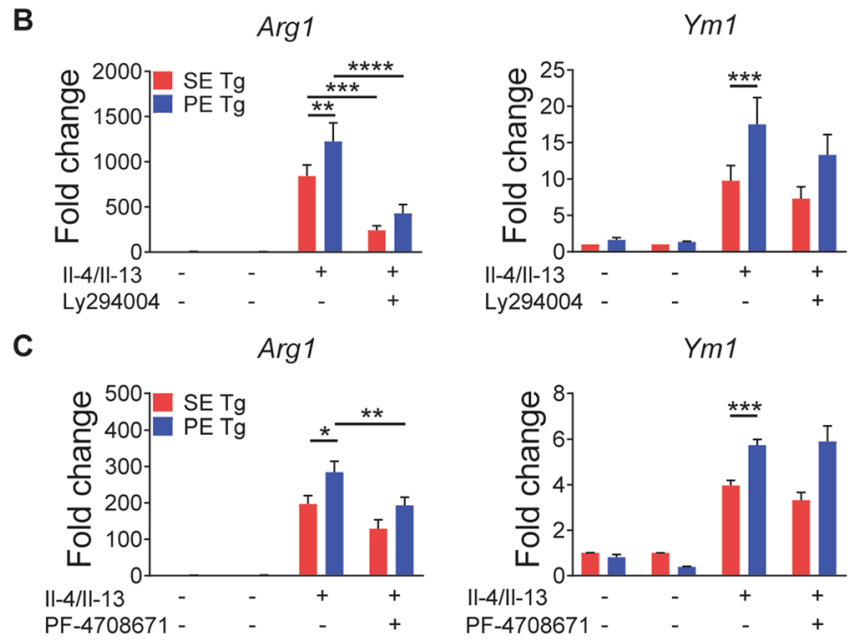

D

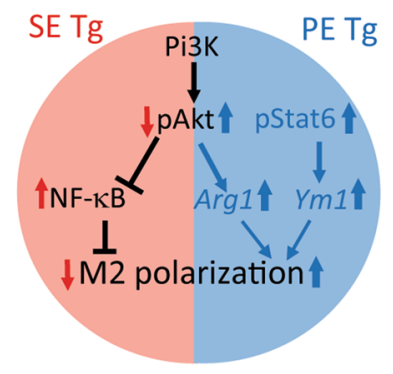

Figure 4. Involvement of signaling pathways in SE Tg and PE Tg BMDMs under M2 polarization conditions. M2 polarization of BMDMs was performed as in Fig. 1. (A) Immunoblotting for pAKt (Ser473) and Akt in BMDMs $10 \mathrm{~min}$ after exposure of cells to M2 polarization conditions. Quantification data represent mean + SEM of 3 independent experiments. (B,C) qPCR analysis for M2 gene markers Arg1 and Ym1 expression in BMDMs pre-treated with Ly294002 (5 $\mu \mathrm{M})(\mathbf{B})$, or PF4708671 $(10 \mu \mathrm{M})(\mathbf{C})$ for $1 \mathrm{~h}$, followed by M2 polarization for $20 \mathrm{~h}$. Data represent mean + SEM of 3 independent experiments. Statistics: within group comparisons, paired $t$ test; between groups comparison, 2-way ANOVA, ${ }^{\star} \mathrm{P}<0.05,{ }^{* *} \mathrm{P}<0.01,{ }^{* * *} \mathrm{P}<0.001,{ }^{* * *} \mathrm{P}<0.0001$. (D) A proposed model of signaling pathway involvement under M2 polarizing conditions.

protein phosphorylation, adaptive immune response and NF- $\kappa$ B (Fig. 5F, Data File S1D). Conversely, GO terms for genes downregulated by $65-79 * \mathrm{PE}$ included bone mineralization and resorption, known as important effector mechanisms in RA pathogenesis. In summary, under M1 polarizing conditions, the PE-expressing TAHR activated an anti-inflammatory or anti-RA transcriptome, whereas the SE-expressing TAHR activated a proinflammatory, pro-RA transcriptome.

To determine whether TAHR polarizing effects could be found in human cells as well, we performed RNAseq analysis in human THP-1 macrophages under M1 polarizing conditions, and found remarkable similarities between the two species (Supplemental Fig. S4, Data File S3). For example, 100 (22\%) of the 446 DEGs upregulated by $65-79 *$ SE in human THP-1 cells were also upregulated by this TAHR in mouse RAW 264.7 macrophages (Supplemental Fig. S4A, Data File S3A,B). Moreover, the top-ranked 60 DEGs that were found to be upregulated by $65-79 *$ SE in both species were searched in PubMed, and $68 \%$ of them were found to be positively associated with RA disease risk or pathogenesis (Supplemental Fig. S4B, Supplemental Table S1, Data File S3B). GO analysis revealed many shared biologic processes (Supplemental Fig. S4C, Data File S3C). Moreover, Kyoto Encyclopedia of Genes and Genomes (KEGG) pathways analysis (Supplemental Fig. S4D, Data File S3C) revealed a high level of correspondence between mouse and human macrophages in many relevant pathways, and identified the KEGG term Rheumatoid Arthritis as the top-ranked pathway in the THP-1 list.

A markedly different transcriptional landscape was observed under M2-polarizing conditions (Fig. 6, Data File S4). Notable amongst the unique upregulated genes by $65-79^{\star} \mathrm{PE}$ in M2-polarizing conditions (Fig. 6C) were anti-angiogenic (Glrx, Col7a1, Rras), anti-inflammatory (Anxa1, Rgs2, Tnfrsf17), anti-bone remodeling 
A

\section{Upregulated DEGs Downregulated DEGs}

65-79*SE

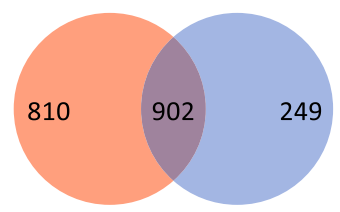

65-79*PE
$65-79 *$ SE

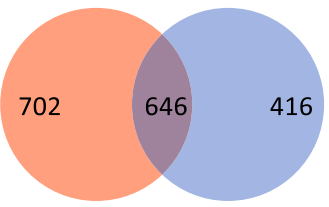

65-79*PE
D

\section{Upregulated GO terms Downregulated GO terms}

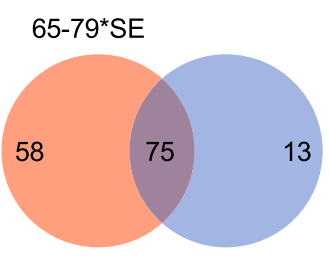

$65-79 * P E$

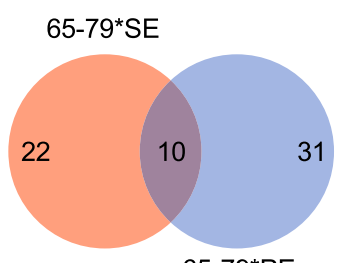

$65-79 * P E$
B

65-79*SE
Unique DEGs

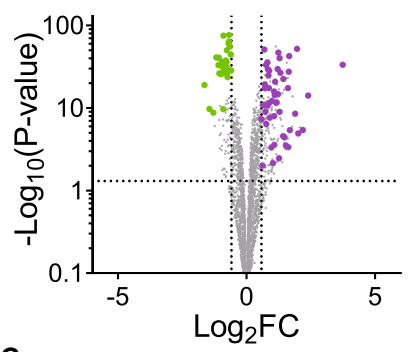

c

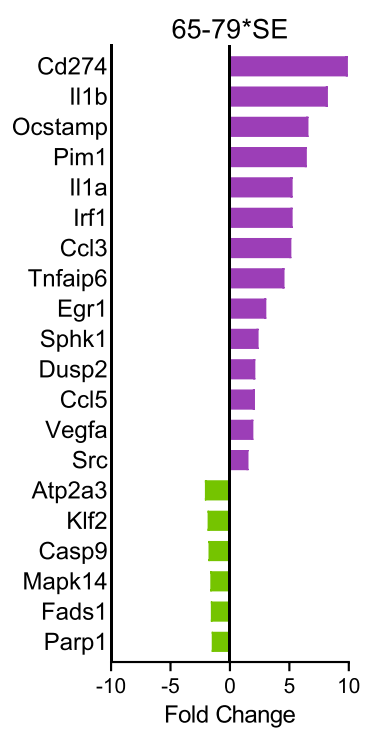

65-79*PE Unique DEGs
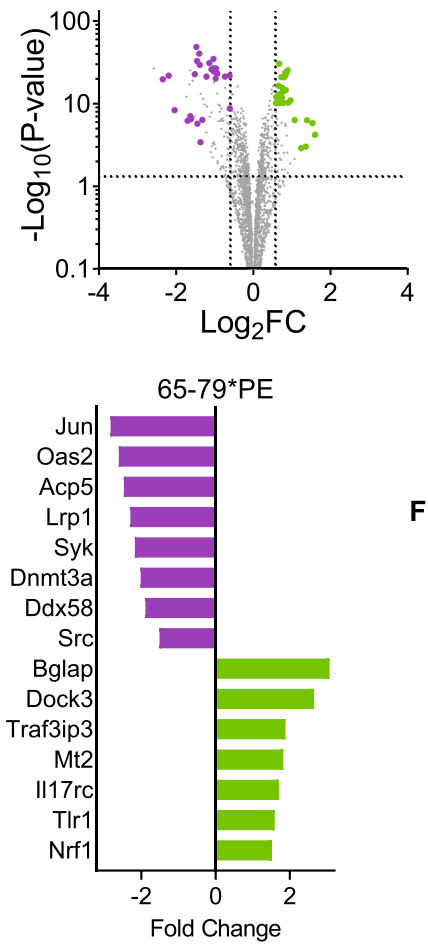

F
E

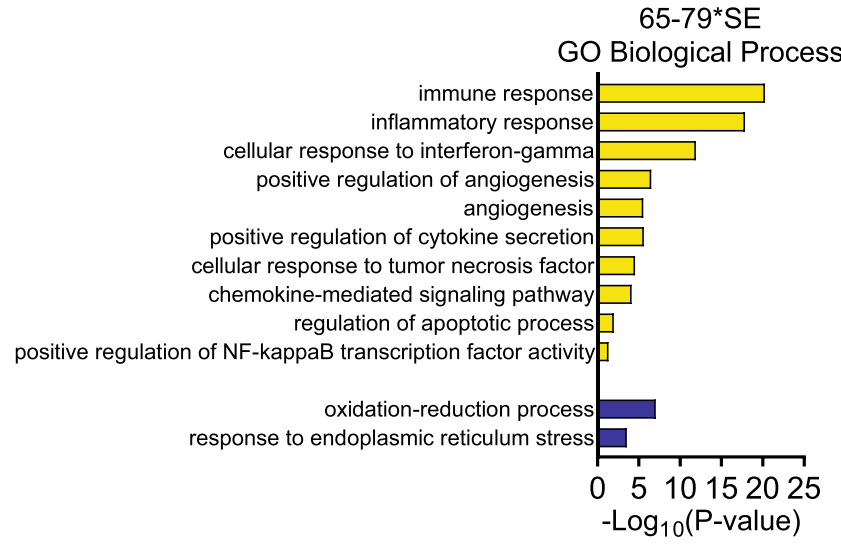

65-79*PE

GO Biological Process

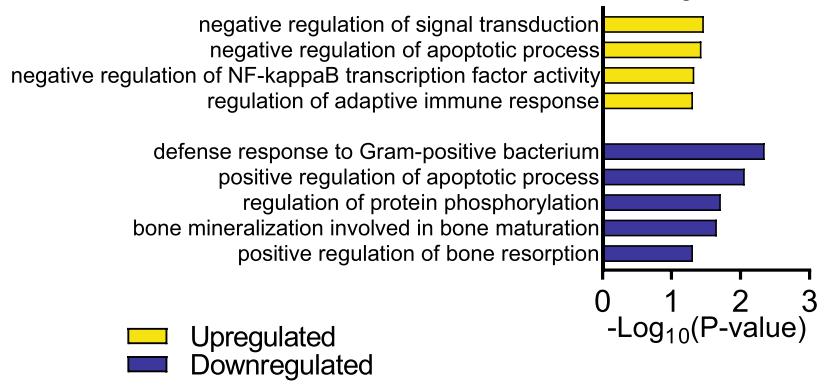

Figure 5. Transcription modulation by $65-79^{\star} \mathrm{SE}$ and $65-79^{\star} \mathrm{PE}$ under $\mathrm{M} 1$ polarizing conditions. Mouse RAW 264.7 macrophages were incubated for 3 days with IFN $\gamma(5 \mathrm{ng} / \mathrm{ml})$ in the presence or absence of $100 \mu \mathrm{g} /$ $\mathrm{ml} 65-79^{\star} \mathrm{SE}$ or $65-79^{\star} \mathrm{PE}$ and RNA-seq analysis was performed on isolated RNA. Data are from 6 biological replicates in 2 independent experiments. (A) Venn diagrams showing upregulated and downregulated DEGs (P adjusted $<0.05$, fold change $>1.5$ ). (B) Volcano plots for unique DEGs for $65-79 \star \mathrm{SE}$ or $65-79 \star \mathrm{PE}$. Purple dots denote pro-RA-associated genes; green dots denote RA-protective genes. (C) Selected notable genes unique for $65-79 * \mathrm{SE}$ or $65-79 * \mathrm{PE}$ with known RA-related functions. Purple bars denote pro-RA-associated genes; green bars denote RA-protective genes. (D) Venn diagrams of overlapping and unique GO terms derived from upregulated and downregulated DEGs in (A). (E, F) Selected GO Biologic Processes for up- and downregulated DEGs by $65-79 * \mathrm{SE}(\mathbf{E})$ or $65-79 * \mathrm{PE}(\mathbf{F})$.

(Ctss), NF- $\kappa$ B inhibitor (Anxa1, Spn), and activator of the Pi3K-Akt pathway (Rras) genes. Downregulated genes in these conditions included many pro-RA genes such as Tnf, Fcrl1, Cxcl10, Il21r, Cxcl2, Ifi44l, Myo1d, among others (Fig. 6A-C, Supplemental Table S1, Data File S4A). In spite of the anti-inflammatory effects by IL-4 $4^{50,61}$, $65-79 *$ SE was able to upregulate various RA and pro-inflammatory genes (e.g. Adamtsl5, Pyr1, Mmp9, Cd74, Cd84 and Lat) and downregulate several anti-RA, anti-inflammatory, or pro-M2 genes (e.g. Rnase4, Col18a1S$t k 17 b, C d 276, C x 3 c r 1)$, although these $65-79 *$ SE effects were weaker than its effect in M1-polarizing conditions (Fig. 6A-C, Supplemental Table S1, Data File S4B). GO analysis (Fig. 6D-F) showed that pathways related to immunity and inflammation were upregulated by $65-79^{\star} \mathrm{SE}$ and downregulated by $65-79^{\star} \mathrm{PE}$ (Fig. 6E,F, Data Files S4C,D). Thus, under M2 polarizing conditions $65-79 * \mathrm{PE}$ and $65-79^{\star} \mathrm{SE}$ had reciprocal effects; the former 
A

Upregulated DEGs

65-79*SE

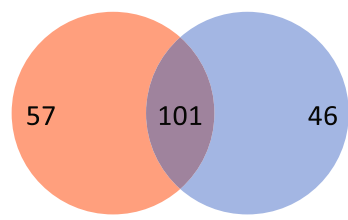

65-79*PE
Downregulated DEGs

65-79*SE

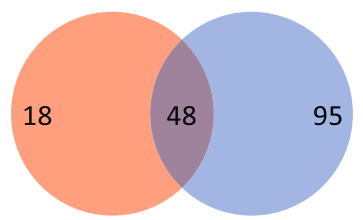

65-79*PE

D Upregulated GO terms Downregulated GO terms

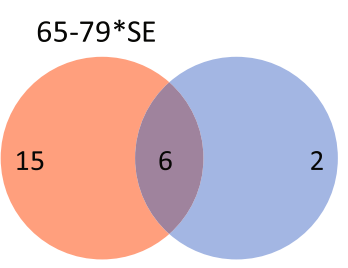

65-79*PE
65-79*SE

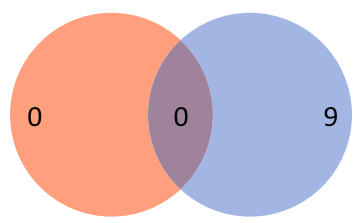

65-79*PE

B

$65-79^{*} S E$

Unique DEGs

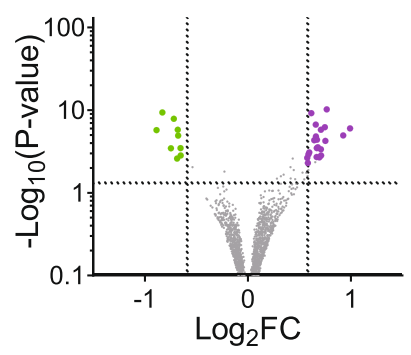

C

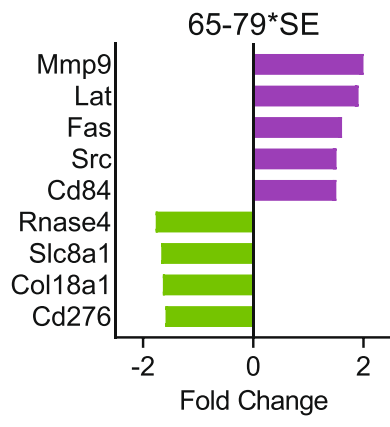

65-79*PE Unique DEGs

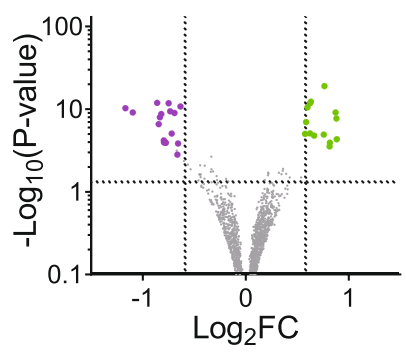

E regulation of macrophage activation cellular response to interferon-gamma

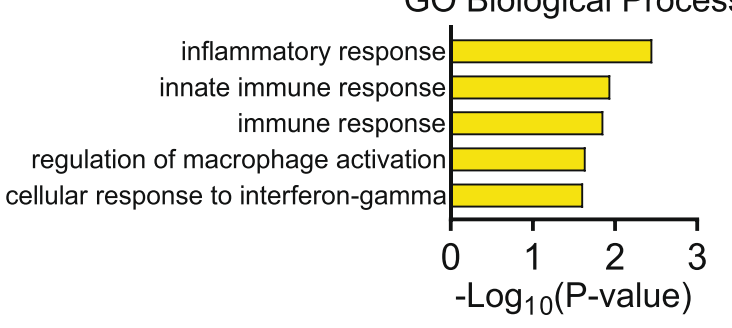

$65-79 *$ SE

65-79*PE GO Biological Process

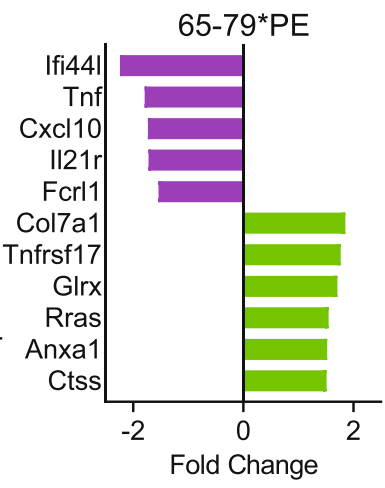
antigen processing and presentation of peptide antigen

negative regulation of $\mathrm{T}$ cell proliferation

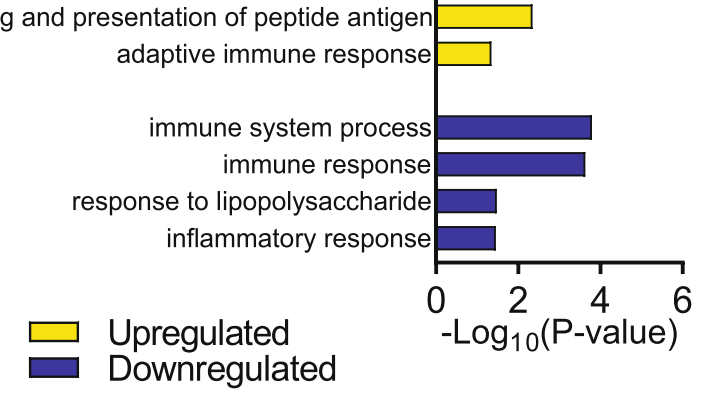

Downregulated

Figure 6. Transcription modulation by $65-79^{\star} \mathrm{SE}$ and $65-79 * \mathrm{PE}$ under M2 polarizing conditions. Mouse RAW 264.7 macrophages were incubated for 3 days with Il-4 $(5 \mathrm{ng} / \mathrm{ml})$ in the presence or absence of $65-79^{\star} \mathrm{SE}$ or $65-79^{\star} \mathrm{PE}$ and RNA-seq analysis was performed as in Fig. 5. Data are from 6 biological replicates in 2 independent experiments. (A) Venn diagrams showing upregulated and downregulated DEGs ( $P$ adjusted $<0.05$, fold change $>1.5$ ). (B) Volcano plots for unique DEGs for $65-79 * \mathrm{SE}$ or $65-79^{\star} \mathrm{PE}$. Purple dots denote pro-RA-associated genes; green dots denote RA-protective genes. (C) Selected notable genes unique for $65-79^{\star} \mathrm{SE}$ or $65-79^{\star} \mathrm{PE}$ with known RA-related functions. Purple bars denote pro-RA-associated genes; green bars denote RA-protective genes. (D) Venn diagrams of overlapping and unique GO terms derived from upregulated and downregulated DEGs in (A). (E,F) Selected GO Biologic Processes for up- and downregulated DEGs by $65-79 * \mathrm{SE}(\mathrm{E})$ or $65-79 * \mathrm{PE}(\mathbf{F})$.

enhanced anti-inflammatory effects, whereas the latter was capable of moderately enhancing an inflammatory transcription profile.

Upstream regulators. To assess the underlying mechanisms driving the gene expression patterns that are induced by $65-79^{\star} \mathrm{SE}$ and $65-79^{\star} \mathrm{PE}$, we performed upstream regulator analyses. In M1-polarizing conditions (Fig. 7A-C, Supplemental Table S2, Data File S5A), $65-79^{\star}$ SE was predicted to stimulate activation of proinflammatory, pro-RA upstream regulators, such as Stat3, Rel, Rela, Jun, Ctnnb1 and Hif1a, among others, and to inhibit anti-arthritis or anti-inflammatory regulators (e.g. Klf2, Xbp1, Foxp3) (Fig. 7A). In these conditions, $65-79 * \mathrm{PE}$ was predicted to activate several anti-inflammatory or NF-KB-inhibiting regulators, such as Nfkbiz, Mta1, Tardbp, Xbp1, and inhibit several key transcription factors known to associate with osteoclastogenesis (Mitf, E2f1), angiogenesis (Srebf1, E2f1, Sox11) and inflammation (Srebf1, Keap1), among others (Fig. 7B, Supplemental Table S2, Data File S5B). 
A

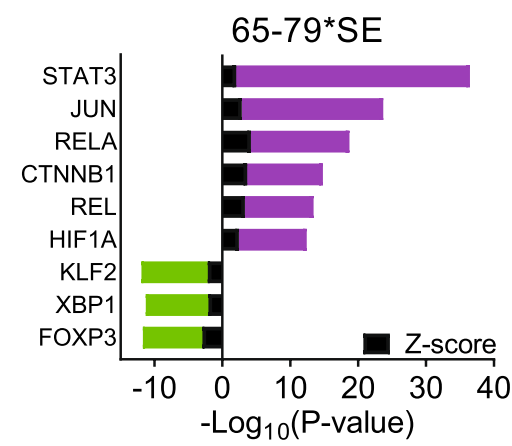

D

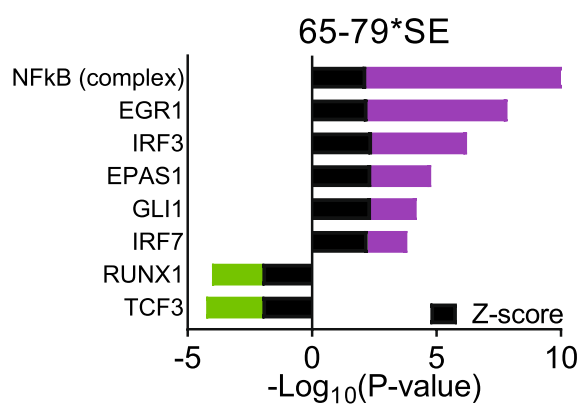

B

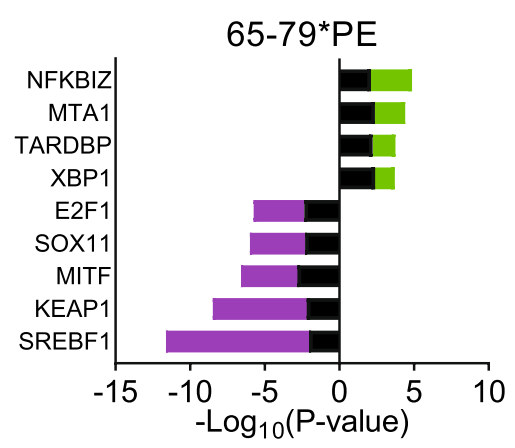

E

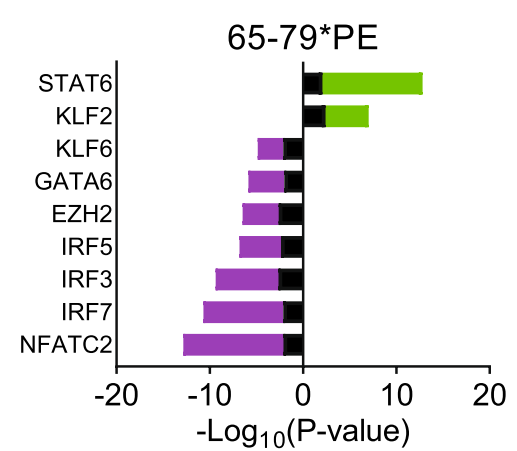

C

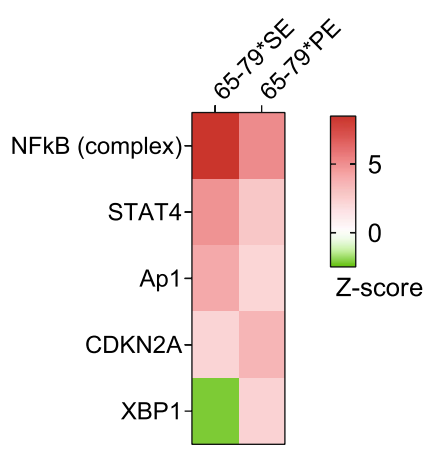

$\mathbf{F}$

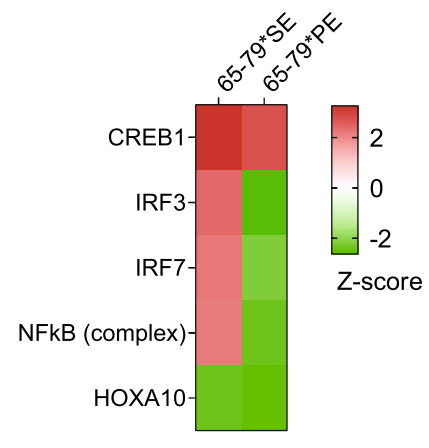

Figure 7. Differential activation of upstream regulators by $65-79 * \mathrm{SE}$ and $65-79 * \mathrm{PE}$ under M1 or M2 polarization conditions. (A,B) Predicted activated and inhibited upstream regulators by $65-79 * \mathrm{SE}(\mathrm{A})$, 65-79*PE (B) in M1-polarizing conditions. (C) Heatmap of notable upstream regulators for $65-79 * \mathrm{SE}$ and $65-79 \star \mathrm{PE}$ in $\mathrm{M} 1$ polarizing conditions. (D,E) Predicted activated and inhibited upstream regulators by 65-79*SE (C), or 65-79*PE (D) in M2-polarizing conditions. In (A,B,D,E); purple bars denote proinflammatory or pro-RA regulators; green bars indicate anti-inflammatory or anti-RA regulators. (F) Heatmap of notable upstream regulators for $65-79^{\star} \mathrm{SE}$ and $65-79^{*} \mathrm{PE}$ in $\mathrm{M} 2$ polarizing conditions. In (C,F); red denotes predicted activation; green denote predicted inhibition.

Under M2 polarizing conditions (Fig. 7D-F, Supplemental Table S2, Data File S5C,D), 65-79*SE was predicted to exert an activation effect on several pro-inflammatory or pro-angiogenic upstream regulators, including Egr1, Irf3, Irf7, Epas1, Gli1 and NF- $\kappa$ B, and an inhibitory effect on anti-RA, anti-osteoclastogenic or antiangiogenic upstream regulators, such as Tcf3 and Runx1 (Fig. 7D). Conversely, 65-79* PE was predicted to activate key M2-inducing and anti-arthritis transcription factors Stat6 and Klf2, and inhibit pro-arthritis (Irf5, Irf7), pro-osteoclastogenic (Nfatc2, Ezh2), pro-angiogenic (Nfatc2, Ezh2, Gata6, Klf6, Irf3), pro-inflammatory (Irf5, Klf6), and pro-M1 (Klf6, Irf3, Irf7) upstream regulators (Fig. 7E). Intriguingly, several upstream regulators were predicted to be modulated in diametrically opposite directions by $65-79 * \mathrm{SE}$ versus $65-79 * \mathrm{PE}$. For example, in M1-polarizing conditions (Fig. 7C), Xbp1, an anti-inflammatory and NF- $\kappa \mathrm{B}$-inhibiting upstream regulator, was predicted to be inhibited by $65-79^{\star}$ SE, yet activated by $65-79^{\star} \mathrm{PE}$. In M2-polarizing conditions (Fig. $7 \mathrm{~F}$ ), a pro-M1 and pro-angiogenic upstream regulator Irf3, and the pro-M1 pro-arthritis upstream regulator Irf7, and $\mathrm{NF}-\kappa \mathrm{B}$ were all predicted to be activated by $65-79 \star \mathrm{SE}$, and reciprocally inhibited by $65-79 \star \mathrm{PE}$. Importantly, consistent with the above findings, and the known role of NF- $\kappa \mathrm{B}$ in the pathogenesis of autoimmune diseases, upstream regulators analysis confirmed a pivotal role for NF- $\kappa B$ complex pathway, with opposite outcomes in the presence of $65-79 *$ SE versus $65-79 * \mathrm{PE}$ (Fig. 8 ).

\section{Discussion}

Decades after MHC-restricted $\mathrm{AP}^{2,3}$ and HLA-disease association (reviewed in ${ }^{1}$ ) were independently discovered, it remains unclear to what extent the two processes are mechanistically-coupled. The findings of this study suggest that aside from their nominal role in AP, which is widely considered an underlying mechanism in HLA-disease association, HLA-DR molecules exhibit allele-specific, AP-independent modulatory effects, which might play a role in autoimmune disease pathogenesis as well.

Given the known pivotal role that macrophages play in regulating pro- and anti-inflammatory events, here we focused on these cells. Our findings revealed differential activation of macrophage polarization pathways by $H L A-D R B 1$ allele-specific gene products. Using primary macrophages derived from two transgenic mouse lines that express distinct human HLA-DR $\beta$ molecules, which differ by only 3 amino acid residues in the TAHR, we observed diametrically opposite polarization patterns. Primary macrophages derived from SE Tg (transgenic mice expressing the SE motif 70-QKRAA-74 in the TAHR of the DR $\beta$ chain) showed strong predilection to 
A

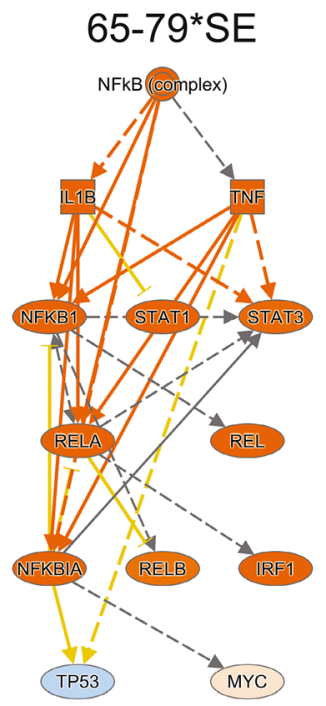

B

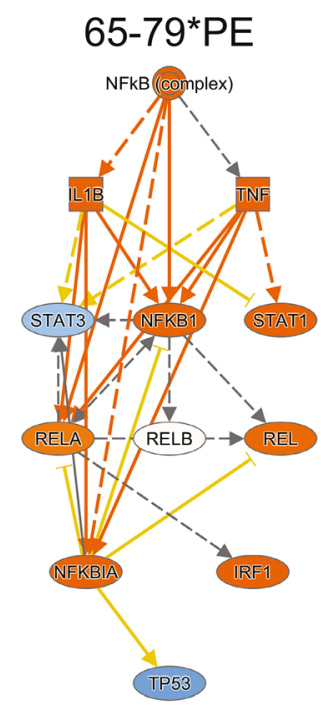

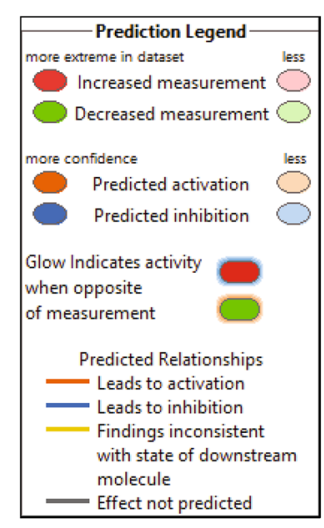

C

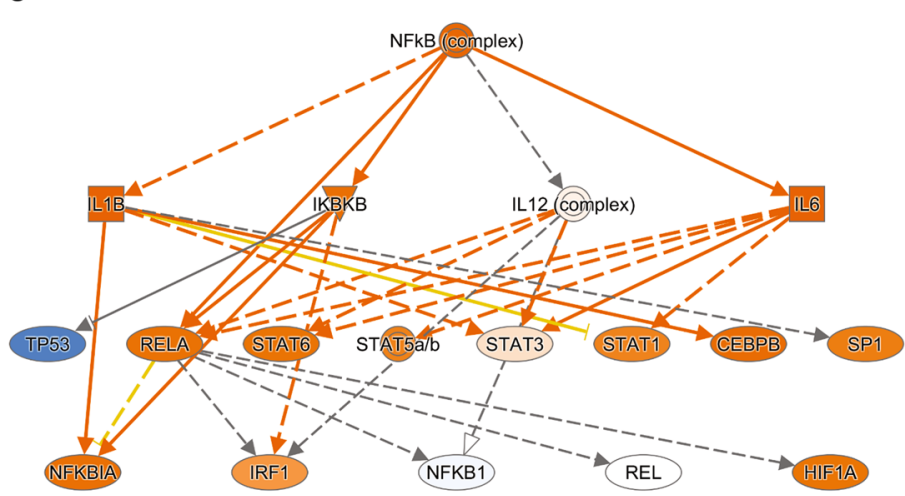

D

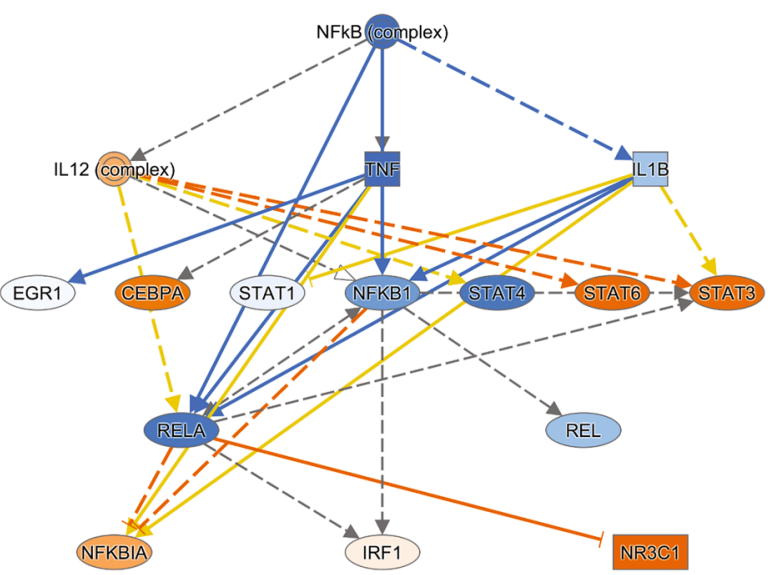

Figure 8. Differential NF- $\kappa B$ pathway activation by $65-79^{\star} \mathrm{SE}$ and $65-79^{\star} \mathrm{PE}$ under M1 (A,B) or M2 (C,D) polarizing conditions. The diagrams were generated through the use of IPA (Qiagen Inc. https://www.qiagenbioi nformatics.com/products/ingenuity-pathway-analysis).

pro-inflammatory M1 macrophage differentiation, while cells derived from PE Tg (expressing a 70-DERAA-74 sequence), displayed preferential differentiation of M2-type macrophages.

The two alleles differentially regulated signaling events. Under M1-polarizing culture conditions, primary macrophages from PE Tg were found to display increased Akt activation and attenuated M1 polarization. In macrophages from SE Tg, on the other hand, SHIP1-mediated Akt inhibition was implicated in enhanced M1 polarization. Under M2-polarizing conditions PE Tg macrophages, but not SE Tg macrophages, selectively enhanced M2 gene expression via the Akt-and possibly Stat6-pathways. Thus, autoimmune disease susceptibility or -protective $H L A-D R B 1$ alleles were found to reciprocally modulate signaling pathways, which determined the efficiency of pro-inflammatory versus anti-inflammatory macrophage differentiation outcomes.

In vitro-differentiated BMDM cultures are devoid of lymphocytes. It is therefore unlikely that the differential macrophage polarization observed here involved AP. To ascertain that AP is indeed not required, and to determine whether the effect could be mapped to the TAHR, we used AP-incompetent 15-mer peptides that correspond to residues 65-79 in the DR $\beta$ chain to explore their transcriptional activation in mouse RAW 264.7 and human THP-1 macrophages. The findings revealed that: (A) synthetic peptides corresponding to TAHRs that differ by only 3 amino acid residues activated allele-specific signature transcriptomes; (B) the effect was APindependent, since it was activated by short, AP-incompetent synthetic peptides; (C) RNA-seq parallels between mouse and human macrophages strengthen the significance of the findings.

Under M1-polarizing conditions, 65-79*SE upregulated the expression levels of many genes that are known to code for pro-inflammatory or known RA disease markers, as well as genes coding for confirmed-or proposedtherapeutic targets, and pathogenic mechanisms, such as osteoclastogenesis, NF-kB activation, angiogenesis, or M1 polarization. Conversely, the SE-expressing TAHR downregulated anti-inflammatory, anti-angiogenesis, inhibitors of NF- $\mathrm{kB}$, and pro-M2 genes. $65-79 * \mathrm{PE}$, on the other hand, upregulated many anti-inflammatory, 
pro-M2 genes, as well as RA-protective, anti-oxidant, genes. Under M2 polarizing conditions, $65-79^{\star} \mathrm{PE}$ downregulated genes and GO processes that are associated with inflammation and RA pathogenesis, while 65-79*SE, notwithstanding the anti-inflammatory tissue culture milieu, managed to upregulate some genes and GO processes known to mediate pro-inflammatory and macrophage activation events.

Importantly, upstream regulator analysis based on gene expression patterns predicted the NF- $\mathrm{KB}$-mediated pathway to be activated by the SE and inhibited by the PE (Fig. 8). This finding is congruent with the key role that NF- $\kappa$ B plays in autoimmune diseases ${ }^{62-67}$. Thus, consistent with their reciprocal impacts on autoimmune disease protection versus susceptibility, the two epitopes dampen (PE) or enhance (SE) pro-inflammatory events.

An RA-protective effect of 70-DERAA-74-coding HLA-DRB1 alleles has been known for some time ${ }^{29}$, but the mechanism underlying this effect has been unclear. A recent study proposed that antigenic mimicry between vinculin and bacterial proteins presented by HLA-DQ molecules, which are commonly associated with SE-coding $H L A-D R B 1$ alleles through linkage disequilibrium may be involved in RA ${ }^{68}$. However, PE-coding HLA-DRB1 alleles have a protective effect in SE-negative individuals as well ${ }^{69}$. Moreover, as discussed above, in addition to their protective effect in RA, PE-coding HLA-DRB1 alleles have been found to decrease disease risk in many other autoimmune conditions that do not share putative antigens with RA. We propose that allele-specific immune modulatory effects of the kind identified here could be considered as an alternative mechanism in HLA-disease associations that are difficult to explain by AP alone.

It is worth mentioning that based on imputation of genomics data it has been suggested by others that in addition to the 5 residues 70-74 in the TAHR that determine the SE-associated RA disease risk, peptide-binding groove residues 11 and 13 associate significantly with RA risk as well ${ }^{70}$, indirectly suggesting that AP may be involved. However, this imputation-based theory has not yet been experimentally validated. Be that as it may, the mechanism proposed here does not exclude involvement of AP in HLA-associated diseases; it offers explanation to aspects of HLA-disease associations that are inconsistent with AP alone. It is not inconceivable that while presentation of specific antigen(s) may determine the anatomic site(s) involved, the SE and PE polarize the immune response to pro- versus anti-inflammatory modes, thereby shaping the pathogenic outcomes.

Our findings lend support to the MHC Cusp theory, which posits that in addition to their nominal function as antigen presenting receptors, MHC molecules express cusp-region epitopes, which activate evolutionaryconserved, non-MHC receptor-mediated pathways ${ }^{10}$. Whereas cell surface calreticulin has been identified as the SE-interacting receptor ${ }^{52,54}$, the identity of the PE-binding partner is presently unknown. However, the parallels between mouse and human cells observed here suggest that, consistent with the MHC Cusp theory, the PE conceivably activates an evolutionary conserved pathway ${ }^{10}$.

This study has focused on two particular DRB1 alleles known to reciprocally modulate disease risk in RA. Further research is required to determine if the SE and PE represent a unique case, or whether other HLA molecules express disease-modulating cusp-region epitopes as well.

\section{Methods}

Mice. Transgenic mice, expressing the human $H L A-D R 4^{\star} 04: 01$ or $H L A-D R 4^{\star} 04: 02$ alleles ${ }^{43,44}$ were kindly provided by Dr. Chela David, at the Mayo Clinic, and are referred to as SE Tg and PE Tg, respectively. The two mouse strains have a mixed (predominantly B6) genetic background and are approximately $99 \%$ identical. Both SE Tg and PE Tg express HLA-DR molecules on BMDMs and splenocytes, without statistically significant expression level differences between the two Tg mouse lines (Supplemental Fig. S5). 10-12 week old male mice were housed under specific pathogen-free and temperature-controlled $\left(25^{\circ} \mathrm{C}\right)$ conditions in a 12 -h dark/light cycle. All experimental mouse protocols were approved by the University of Michigan Unit for Laboratory Animal Medicine and by the University of Michigan Committee on Use and Care of Animals. All applicable federal, state, local, and institutional laws, regulations, policies, and standards governing animal research were followed. In some experiments, LPS $(500 \mu \mathrm{g} / \mathrm{kg})$ was administered to $10-12$ week old male mice using a single i.p. injection. Serum or peritoneal exudate cells (PECs) were collected 4 h. after i.p. injection.

Reagents. All reagents used, along with vendor names and catalog numbers are listed in Supplemental Table S3.

Synthetic peptides, purchased from Bioworld (Dublin, Ohio, USA) were 95-99\% pure. For additional quality assurance, we periodically perform Maldi-TOF Mass Spectrometry and NMR analyses to rule out impurities.

Primary macrophage culture conditions. Primary mouse BMDMs were isolated and cultured as previously described ${ }^{56}$. Peritoneal macrophages were isolated by injecting ice-cold PBS into the peritoneal cavity. PECs were subsequently collected, centrifuged and washed once with PBS. For qRT-PCR experiments, macrophages were cultured for 3 days in 6-well plates $\left(2 \times 10^{6}\right.$ cells per well) in a-MEM with $10 \%$ (v/v) FBS, $100 \mathrm{U} / \mathrm{ml}$ penicillin and $100 \mu \mathrm{g} / \mathrm{ml}$ streptomycin, along with $10 \mathrm{ng} / \mathrm{ml}$ recombinant macrophage colony stimulating factor (M-CSF). Culture media were refreshed daily. In cell function experiments, instead of M-CSF, macrophages were cultured for 3 days in $20 \%$ (v/v) L929 cell conditioned media, in addition to $0.5 \%$ (v/v) pyruvate, $10 \%$ (v/v) FBS, $100 \mu \mathrm{g} / \mathrm{ml}$ streptomycin and $100 \mathrm{U} / \mathrm{ml}$ penicillin. To induce M1 polarization, cells were treated with $1 \mathrm{ng} / \mathrm{ml} \mathrm{LPS}$ and $20 \mathrm{ng} / \mathrm{ml} \mathrm{IFN} \gamma$ for $24 \mathrm{~h}$. To induce M2 polarization, Il-4 and Il-13 (10 ng/ml each) were added for $24 \mathrm{~h}$. For experiments with inhibitors, cells were treated with Ly294004 (5 $\mu \mathrm{M})$, PF-4708671 $(10 \mu \mathrm{M})$, or wedelolactone $(10 \mu \mathrm{M})$ for $1 \mathrm{hr}$ prior to polarization.

Macrophage cell lines culture conditions. Mouse RAW 264.7 macrophages were maintained in DMEM containing $10 \%(\mathrm{v} / \mathrm{v}) \mathrm{FBS}, 100 \mu \mathrm{g} / \mathrm{ml}$ streptomycin and $100 \mathrm{U} / \mathrm{ml}$ penicillin. Cells were cultured in T75 flasks to confluence and were split every 3 days. THP-1 cells were cultured in a 10\% (v/v) FBS-RPMI 1640 medium 
containing $100 \mathrm{U} / \mathrm{ml}$ penicillin and $100 \mu \mathrm{g} / \mathrm{ml}$ streptomycin. To differentiate THP-1 cells into macrophages they were cultured for 3 days with phorbol 12-myristate 13-acetate (PMA, $85 \mathrm{nM}$ ). Differentiated cells were then cultured without PMA for an additional 5 days prior to the experiments.

RNA isolation and qRT-PCR. Mouse cells were lysed with Trizol for RNA isolation. Direct-Zol ${ }^{\mathrm{Ts}}$ RNA miniprep (Zymo research) was used to isolate total RNA. Isolation of RNA from THP-1 cells was carried out with a RNeasy Plus Mini kit (Qiagen). The High-Capacity cDNA Reverse Transcription Kit (Applied Biosystems) was used to synthesize cDNA. qRT-PCR was performed by Fast SYBRTM Green Master Mix (Applied Biosystems), with sets of primers as listed in Supplementary Table S4, using a StepOnePlus Real-Time PCR system (Applied Biosystems). A StepOne Software was used to analyze the data with the $\Delta \Delta \mathrm{CT}$ method.

Immunoblots. After being washed with ice-cold PBS, cells were lysed in RIPA buffer (Sigma) with EDTAfree protease phosphatase inhibitors (Roche Diagnostics). Protein concentration in lysates was determined using the DC protein assay (BioRad). Proteins were loaded onto 4-20\% SDS-PAGE gels (Invitrogen), and after electrophoretic separation, transferred to nitrocellulose membranes (BioRad), followed by incubation with appropriate primary and secondary antibodies. All primary antibodies were diluted 1:1000 in 5\% (v/v) BSA (SigmaAldrich), except $\beta$-Actin (1:4,000). The primary antibodies used were anti: Akt (\#9272), pAkt (S473, \#9271), STAT6 (\#9362), pSTAT6 (Y641, \#56554), SHIP1 (\#2728), pSHIP1 (Y1020, \#3941), PTEN (\#9559), pPTEN (S380/T382/383, \#9549) (all from Cell Signaling Technology), or anti- $\beta$-Actin (Invitrogen, BA3R). Second-stage antibodies included Anti-Rabbit IgG HRP-conjugated (Cell Signaling Technology, 1:1000) or anti-mouse IgG HRP-conjugated (GE Healthcare, 1:8000). All blots, except those in Supplementary Fig. S1B, were stripped and re-hybridized as one piece. In Supplementary Fig. S1B, membranes were first hybridized with an anti-phoshoSHIP1 antibody, then stripped, blocked and the top part was cut and hybridized with anti-total SHIP1 antibody, while the bottom part was hybridized with anti- $\beta$-Actin antibody. Band visualization was performed using a SuperSignal West Pico Plus ECL substrate (Thermo Scientific) and an Omega Lum C imaging system (Gel Company). Band quantification was performed in duplicates with the ImageJ software.

Cytokine measurements. Tnfa, Il-12p70, Il-10 and Il-6 were quantified in culture supernatants by ELISA kits (R\&D). Serum cytokine levels were measured using Quantibody Mouse Cytokine array 1 (RayBiotech). Slides were scanned by RayBiotech Service Department and data were analyzed using a RayBiotech Mouse Cytokine Array 1 software (QAM-CYT-1-SW). Sample concentrations were derived from mean fluorescence intensities, relative to standard curves, generated using the manufacturer's standards.

NO and arginase assays. NO production was quantified using the fluorescent NO dye 4,5-diaminofluorescein diacetate (DAF-2DA) as previously described ${ }^{71}$. Cells were first plated overnight in flat-bottom, 96-well plates, then washed with DMEM/phenol red-free medium (Sigma). Cultures were then loaded with $20 \mu \mathrm{M}$ DAF$2 \mathrm{DA}$ at $37^{\circ} \mathrm{C}$ for $1 \mathrm{~h}$. in the dark. Fluorescence levels were recorded every 5 min over a period of $500 \mathrm{~min}$ using a Synergy H1 hybrid reader system (Biotek) at excitation/emission wavelengths of $488 / 515 \mathrm{~nm}$. NO production rates are expressed as fluorescence units (FU) per minute. Measurement of arginase activity was carried out using the Arginase Activity Colorimetric Assay Kit (Biovision).

RNA-Seq. Cells were incubated with or without IFN $\gamma(5 \mathrm{ng} / \mathrm{ml})$ or Il-4 $(5 \mathrm{ng} / \mathrm{ml})$ in the presence or absence of 15 -mer peptides $65-79^{\star} \mathrm{SE}$ or $65-79^{\star} \mathrm{PE}(100 \mu \mathrm{g} / \mathrm{ml})$ and medium was refreshed at $48 \mathrm{~h}$. At $72 \mathrm{~h}$., total RNA was isolated using the Direct-Zol ${ }^{\mathrm{m}} \mathrm{RNA}$ miniprep (Zymo research). Genomic DNA was removed using the Turbo DNA-Free kit (Ambion). Total RNA concentration and integrity were determined with an Agilent Bioanalyzer (Agilent). All RNA samples had an integrity number of 7.5 or higher. Two hundred ng of total RNA were used to generate libraries using the TruSeq Stranded mRNA Sample Preparation Kit (Illumina). Single-end reads of $100 \mathrm{bp}$ for each sample were produced with Illumina's HiSeq2500v4 instrument. Raw counts were attained using featureCounts from the Rsubread1.5.0p3 package and Gencode-M12 gene annotations using only uniquely aligned reads. For THP-1, Raw counts were attained using featureCounts from the Rsubread-1.6.1 package and -Gencode28-hg38 gene annotations using only uniquely aligned reads. DESeq2-1.16.1 within R-3.4.1 was used to perform data normalization and differential expression analysis with an adjusted p value threshold of 0.05. Mouse annotations were verified using the MGI database (http://www.informatics.jax.org/index.shtml) ${ }^{72}$ and only protein encoding genes were considered. Genes with a fold-change of $>1.5$ and $p$ values of 0.05 were used for GO term analysis. RA relevant genes were identified based on published literature. The DAVID bioinformatics database (version 6.8) 73 $^{73}$ was used for GO analysis, using an Expression Analysis Systematic Explorer (EASE) score threshold of 0.1 for detection of gene enrichment. GO terms with an FDR of $5 \%$ were considered significant. To identify unique GO terms, significant GO terms obtained in 65-79*SE-stimulated cells in either M1- or M2-polarizing culture conditions were compared to those obtained in $65-79 * \mathrm{PE}$-stimulated cells under the same polarizing culture conditions.

Upstream regulator analysis. Ingenuity Pathway Analysis (QIAGEN Inc., CA, www.ingenuity.com/ index.html) was used to perform upstream regulator analysis. Genes differentially expressed in the presence of $65-79^{\star} \mathrm{SE}$ or $65-79^{\star} \mathrm{PE}$ were analyzed to identify transcriptional regulators that may be responsible for the gene expression changes observed. Only transcriptional regulator relationships observed using experimental data were used in this analysis. A z score was used to examine the robustness of predicted activation or inhibition of specific transcription regulators based on enrichment and differential expression (upregulation or downregula- 
tion) in the presence of $65-79 *$ SE or $65-79 *$ PE. $p$ values $<0.05$ by Fisher's Exact Test after correction for multiple testing were considered significant.

Flow cytometry. BMDMs were grown in aMEM with M-CSF (10 ng/ml) for 4 days. Splenocytes were isolated as single-cell suspensions by mashing spleen tissues through a $70 \mu \mathrm{m}$ cell strainer. Red blood cells were lysed using an RBC lysis buffer (Biolegend) according the manufacturer's instructions. Non-permeabilized BMDMs or splenocytes were incubated for $30 \mathrm{~min}$ on ice with a phycoerythrin-conjugated anti-HLA-DR antibody (Clone L243, Biolegend) and Ghost Dye Violet 510 (Cat\# 13-0870-T100, Tonbo). Cells were analyzed using a ZE5 flow cytometer (Bio-Rad). Histograms were generated using the FlowJo software, version 10.7.1.

Statistics. Data are shown as the mean and SEM calculated using a GraphPad Prism Software (version 7). Unless otherwise specified, a 2-way ANOVA was used to determine significance. $p$ values $<0.05$ were considered significant. Bar graphs were generated using GraphPad Prism version 7.0.0 from GraphPad Software, CA, www. graphpad.com.

\section{Data availability}

The RNA-seq data have been deposited in NCBI's Gene Expression Omnibus and are accessible through GEO accession number GSE159821.

Received: 14 December 2019; Accepted: 18 January 2021

Published online: 28 January 2021

\section{References}

1. Ryder, L. P., Svejgaard, A. \& Dausset, J. Genetics of HLA disease association. Annu. Rev. Genet. 15, 169-187 (1981).

2. Doherty, P. C. \& Zinkernagel, R. M. H-2 compatibility is required for T-cell-mediated lysis of target cells infected with lymphocytic choriomeningitis virus. J. Exp. Med. 141, 502-507 (1975).

3. Zinkernagel, R. M. \& Doherty, P. C. Restriction of in vitro T cell-mediated cytotoxicity in lymphocytic choriomeningitis within a syngeneic or semiallogeneic system. Nature 248, 701-702 (1974).

4. Nepom, G. T. \& Kwok, W. W. Molecular basis for HLA-DQ associations with IDDM. Diabetes 47, 1177-1184 (1998).

5. Oldstone, M. B. Molecular mimicry and immune-mediated diseases. FASEB J. 12, 1255-1265 (1998).

6. Ridgway, W. M. \& Fathman, C. G. The association of MHC with autoimmune diseases: Understanding the pathogenesis of autoimmune diabetes. Clin. Immunol. Immunopathol. 86, 3-10 (1998).

7. Yin, L. et al. Recognition of self and altered self by T cells in autoimmunity and allergy. Protein Cell 4, 8-16 (2013).

8. Koning, F., Thomas, R., Rossjohn, J. \& Toes, R. E. Coeliac disease and rheumatoid arthritis: Similar mechanisms, different antigens. Nat. Rev. Rheumatol. 11, 450-461 (2015).

9. Ilonen, J., Lempainen, J. \& Veijola, R. The heterogeneous pathogenesis of type 1 diabetes mellitus. Nat. Rev. Endocrinol. 15, 635-650 (2019).

10. de Almeida, D. E. \& Holoshitz, J. MHC molecules in health and disease: At the cusp of a paradigm shift. Self Nonself 2, 43-48 (2011).

11. Holoshitz, J. The quest for better understanding of HLA-disease association: Scenes from a road less travelled by. Discov. Med. 16, 93-101 (2013).

12. Bowness, P. Hla-B27. Annu. Rev. Immunol. 33, 29-48 (2015).

13. Ranganathan, V., Gracey, E., Brown, M. A., Inman, R. D. \& Haroon, N. Pathogenesis of ankylosing spondylitis-recent advances and future directions. Nat. Rev. Rheumatol. 13, 359-367 (2017).

14. Schittenhelm, R. B., Sian, T. C., Wilmann, P. G., Dudek, N. L. \& Purcell, A. W. Revisiting the arthritogenic peptide theory: Quantitative not qualitative changes in the peptide repertoire of HLA-B27 allotypes. Arthritis Rheumatol. 67, 702-713 (2015).

15. Navid, F. \& Colbert, R. A. Causes and consequences of endoplasmic reticulum stress in rheumatic disease. Nat. Rev. Rheumatol. 13, 25-40 (2017)

16. Gregersen, P. K., Silver, J. \& Winchester, R. J. The shared epitope hypothesis. An approach to understanding the molecular genetics of susceptibility to rheumatoid arthritis. Arthritis Rheum. 30, 1205-1213 (1987).

17. Tait, B. D., Drummond, B. P., Varney, M. D. \& Harrison, L. C. HLA-DRB1 ${ }^{\star} 0401$ is associated with susceptibility to insulindependent diabetes mellitus independently of the DQB1 locus. Eur. J. Immunogenet. 22, 289-297 (1995).

18. Doherty, D. G. et al. Allelic sequence variation in the HLA class II genes and proteins in patients with autoimmune hepatitis. Hepatology 19, 609-615 (1994).

19. Haworth, S. et al. Polymyalgia rheumatica is associated with both HLA-DRB1*0401 and DRB1*0404. Br. J. Rheumatol. 35, 632-635 (1996).

20. Martinez-Taboda, V. M. et al. HLA-DRB1 allele distribution in polymyalgia rheumatica and giant cell arteritis: Influence on clinical subgroups and prognosis. Semin. Arthritis Rheum. 34, 454-464 (2004).

21. Hollenbach, J. A. et al. A specific amino acid motif of HLA-DRB1 mediates risk and interacts with smoking history in Parkinson's disease. Proc. Natl. Acad. Sci. USA 116, 7419-7424 (2019).

22. Gehlot, P., Volk, S. L., Rios, H. F., Jepsen, K. J. \& Holoshitz, J. Spontaneous destructive periodontitis and skeletal bone damage in transgenic mice carrying a human shared epitope-coding HLA-DRB1 allele. RMD Open 2, e000349 (2016).

23. Bonfil, J. J. et al. A "case control" study on the role of HLA DR4 in severe periodontitis and rapidly progressive periodontitis. Identification of types and subtypes using molecular biology (PCR. SSO). J. Clin. Periodontol. 26, 77-84 (1999).

24. Marotte, H. et al. The association between periodontal disease and joint destruction in rheumatoid arthritis extends the link between the HLA-DR shared epitope and severity of bone destruction. Ann. Rheum. Dis. 65, 905-909 (2006).

25. Chan, M. T. et al. Associations of erosive arthritis with anti-cyclic citrullinated peptide antibodies and MHC Class II alleles in systemic lupus erythematosus. J. Rheumatol. 35, 77-83 (2008).

26. Korendowych, E., Dixey, J., Cox, B., Jones, S. \& McHugh, N. The influence of the HLA-DRB1 rheumatoid arthritis shared epitope on the clinical characteristics and radiological outcome of psoriatic arthritis. J. Rheumatol. 30, 96-101 (2003).

27. Scholz, E. et al. A comparative analysis of the peptide repertoires of HLA-DR molecules differentially associated with rheumatoid arthritis. Arthritis Rheumatol. 68, 2412-2421 (2016).

28. Carrier, N. et al. The DERAA HLA-DR alleles in patients with early polyarthritis: Protection against severe disease and lack of association with rheumatoid arthritis autoantibodies. Arthritis Rheumatol. 60, 698-707 (2009).

29. Kampstra, A. S. B. \& Toes, R. E. M. HLA class II and rheumatoid arthritis: The bumpy road of revelation. Immunogenetics 69 , 597-603 (2017). 
30. Seidl, C. et al. Protection against severe disease is conferred by DERAA-bearing HLA-DRB1 alleles among HLA-DQ3 and HLADQ5 positive rheumatoid arthritis patients. Hum. Immunol. 62, 523-529 (2001).

31. van der Woude, D. et al. Protection against anti-citrullinated protein antibody-positive rheumatoid arthritis is predominantly associated with HLA-DRB1*1301: A meta-analysis of HLA-DRB1 associations with anti-citrullinated protein antibody-positive and anti-citrullinated protein antibody-negative rheumatoid arthritis in four European populations. Arthritis Rheumatol. 62, 1236-1245 (2010).

32. Misra, M. K., Damotte, V. \& Hollenbach, J. A. The immunogenetics of neurological disease. Immunology 153, 399-414 (2018).

33. Furukawa, H. et al. The role of common protective alleles HLA-DRB1 ${ }^{\star} 13$ among systemic autoimmune diseases. Genes Immun. 18, 1-7 (2017)

34. Flam, S. T. et al. The HLA profiles of mixed connective tissue disease differ distinctly from the profiles of clinically related connective tissue diseases. Rheumatology (Oxford) 54, 528-535 (2015).

35. Xu, Y. et al. Human leukocyte antigen (HLA)-DRB1 allele polymorphisms and systemic sclerosis. Mod. Rheumatol. 20, 1-8 (2019).

36. Kawasaki, A. et al. Protective role of HLA-DRB1*13:02 against microscopic polyangiitis and MPO-ANCA-positive vasculitides in a japanese population: A case-control study. PLoS One 11, e0154393 (2016).

37. Hor, H. et al. Genome-wide association study identifies new HLA class II haplotypes strongly protective against narcolepsy. Nat. Genet. 42, 786-789 (2010).

38. Tardito, S. et al. Macrophage M1/M2 polarization and rheumatoid arthritis: A systematic review. Autoimmun. Rev. 18, 102397 (2019).

39. Di Benedetto, P., Ruscitti, P., Vadasz, Z., Toubi, E. \& Giacomelli, R. Macrophages with regulatory functions, a possible new therapeutic perspective in autoimmune diseases. Autoimmun. Rev. 18, 102369 (2019).

40. Davies, L. C. et al. Distinct bone marrow-derived and tissue-resident macrophage lineages proliferate at key stages during inflammation. Nat. Commun. 4, 1886 (2013).

41. Udalova, I. A., Mantovani, A. \& Feldmann, M. Macrophage heterogeneity in the context of rheumatoid arthritis. Nat. Rev. Rheumatol. 12, 472-485 (2016)

42. Varga, T. et al. Highly dynamic transcriptional signature of distinct macrophage subsets during sterile inflammation, resolution, and tissue repair. J. Immunol. 196, 4771-4782 (2016).

43. Pan, S., Trejo, T., Hansen, J., Smart, M. \& David, C. S. HLA-DR4 (DRB1 $\left.{ }^{\star} 0401\right)$ transgenic mice expressing an altered CD4-binding site: Specificity and magnitude of DR4-restricted T cell response. J. Immunol. 161, 2925-2929 (1998).

44. Taneja, V. et al. HLA-DRB1*0402 (DW10) transgene protects collagen-induced arthritis-susceptible H2Aq and DRB1*0401 (DW4) transgenic mice from arthritis. J. Immunol. 171, 4431-4438 (2003).

45. Rath, M., Muller, I., Kropf, P., Closs, E. I. \& Munder, M. Metabolism via arginase or nitric oxide synthase: Two competing arginine pathways in macrophages. Front. Immunol. 5, 532 (2014).

46. Vergadi, E., Ieronymaki, E., Lyroni, K., Vaporidi, K. \& Tsatsanis, C. Akt signaling pathway in macrophage activation and M1/M2 polarization. J. Immunol. 198, 1006-1014 (2017).

47. Weichhart, T., Hengstschlager, M. \& Linke, M. Regulation of innate immune cell function by mTOR. Nat. Rev. Immunol. 15, 599-614 (2015).

48. Fong, C. H. et al. An antiinflammatory role for IKKbeta through the inhibition of "classical" macrophage activation. J. Exp. Med. 205, 1269-1276 (2008).

49. Dan, H. C. et al. Akt-dependent regulation of NF-\{kappa\}B is controlled by mTOR and Raptor in association with IKK. Genes Dev. 22, 1490-1500 (2008).

50. Byles, V. et al. The TSC-mTOR pathway regulates macrophage polarization. Nat. Commun. 4, 2834 (2013).

51. Holoshitz, J. et al. An HLA-DRB1-coded signal transduction ligand facilitates inflammatory arthritis: A new mechanism of autoimmunity. J. Immunol. 190, 48-57 (2013).

52. Ling, S., Cheng, A., Pumpens, P., Michalak, M. \& Holoshitz, J. Identification of the rheumatoid arthritis shared epitope binding site on calreticulin. PLoS One 5, e11703 (2010).

53. Ling, S. et al. The rheumatoid arthritis shared epitope increases cellular susceptibility to oxidative stress by antagonizing an adenosine-mediated anti-oxidative pathway. Arthritis Res. Ther. 9, R5 (2007).

54. Ling, S., Pi, X. \& Holoshitz, J. The rheumatoid arthritis shared epitope triggers innate immune signaling via cell surface calreticulin. J. Immunol. 179, 6359-6367 (2007)

55. van Drongelen, V., Ali, W. H. \& Holoshitz, J. Uncovering a shared epitope-activated protein citrullination pathway. J. Immunol. 205, 579-586 (2020).

56. Fu, J. et al. A small shared epitope-mimetic compound potently accelerates osteoclast-mediated bone damage in autoimmune arthritis. J. Immunol. 191, 2096-2103 (2013).

57. Das, P. et al. An HLA-DRB1*0402 derived peptide (HV3 65-79) prevents collagen-induced arthritis in HLA-DQ8 transgenic mice. Hum. Immunol. 60, 575-582 (1999).

58. de Vries, R. R., Huizinga, T. W. \& Toes, R. E. Redefining the HLA and RA association: To be or not to be anti-CCP positive. J. Autoimmun. 25(Suppl), 21-25 (2005).

59. Ling, S. et al. Shared epitope-antagonistic ligands: A new therapeutic strategy in mice with erosive arthritis. Arthritis Rheumatol. 67, 2061-2070 (2015).

60. Mackie, S. L. et al. A spectrum of susceptibility to rheumatoid arthritis within HLA-DRB1: Stratification by autoantibody status in a large UK population. Genes Immun. 13, 120-128 (2012).

61. Hart, P. H. et al. Potential antiinflammatory effects of interleukin 4: Suppression of human monocyte tumor necrosis factor alpha, interleukin 1, and prostaglandin E2. Proc. Natl. Acad. Sci. USA 86, 3803-3807 (1989).

62. Afonina, I. S., Zhong, Z., Karin, M. \& Beyaert, R. Limiting inflammation-the negative regulation of NF-kappaB and the NLRP3 inflammasome. Nat. Immunol. 18, 861-869 (2017).

63. Dorrington, M. G. \& Fraser, I. D. C. NF-kappaB signaling in macrophages: Dynamics, crosstalk, and signal integration. Front Immunol. 10, 705 (2019).

64. Miraghazadeh, B. \& Cook, M. C. Nuclear factor-kappaB in autoimmunity: Man and mouse. Front Immunol. 9, 613 (2018).

65. Chen, Z., Bozec, A., Ramming, A. \& Schett, G. Anti-inflammatory and immune-regulatory cytokines in rheumatoid arthritis. Nat. Rev. Rheumatol 15, 9-17 (2019).

66. Han, Z., Boyle, D. L., Manning, A. M. \& Firestein, G. S. AP-1 and NF-kappaB regulation in rheumatoid arthritis and murine collagen-induced arthritis. Autoimmunity 28, 197-208 (1998).

67. Fu, J. et al. Shared epitope-aryl hydrocarbon receptor crosstalk underlies the mechanism of gene-environment interaction in autoimmune arthritis. Proc. Natl. Acad. Sci. USA 115, 4755-4760 (2018).

68. van Heemst, J. et al. Crossreactivity to vinculin and microbes provides a molecular basis for HLA-based protection against rheumatoid arthritis. Nat. Commun. 6, 6681 (2015).

69. van der Helm-van Mil, A. H. et al. An independent role of protective HLA class II alleles in rheumatoid arthritis severity and susceptibility. Arthritis Rheum. 52, 2637-2644 (2005).

70. Raychaudhuri, S. et al. Five amino acids in three HLA proteins explain most of the association between MHC and seropositive rheumatoid arthritis. Nat. Genet. 44, 291-296 (2012). 
71. Ling, S., Lai, A., Borschukova, O., Pumpens, P. \& Holoshitz, J. Activation of nitric oxide signaling by the rheumatoid arthritis shared epitope. Arthritis Rheum. 54, 3423-3432 (2006).

72. Smith, C. L., Blake, J. A., Kadin, J. A., Richardson, J. E. \& Bult, C. J. Mouse Genome Database (MGD)-2018: knowledgebase for the laboratory mouse. Nucleic Acids Res. 46, D836-D842 (2018).

73. Huang da, W., Sherman, B. T. \& Lempicki, R. A. Systematic and integrative analysis of large gene lists using DAVID bioinformatics resources. Nat. Protoc. 4, 44-57 (2009).

\section{Acknowledgements}

We thank E. Henson, T. Tubo, Y. Liu and Dr. W. H. Ali-Hanel at the University of Michigan for technical assistance, and Dr. P. Gourh and Dr. P. Grayson at the National Institute of Arthritis and Musculoskeletal and Skin Diseases for useful comments on the manuscript.

\section{Author contributions}

J.H. conceived the study. V.D., B.M.S., A.H.S. and J.H. designed the experiments. V.D., S.V.N., and B.M.S. performed the experiments. V.D., B.M.S., A.H.S. and J.H. analyzed data. F.W.M. and J.H. provided funding. V.D. and J.H. wrote the paper. All authors interpreted the data, reviewed the paper, and approved the final version of the manuscript.

\section{Funding}

This work was supported by the Extramural Program of the National Institute of Arthritis and Musculoskeletal and Skin Diseases (Grants R01AR059085, R61AR073014, R33AR073014, R01ARR074930) to J.H., the National Institute of Environmental Health Sciences Extramural Program (Contract HHSN273201600123P) to J.H., and the Intramural Research Program at the National Institute of Environmental Health Sciences (Grant ES101074) to F.W.M. The content is solely the responsibility of the authors and does not necessarily represent the official views of the National Institutes of Health.

\section{Competing interests}

J.H. is an Inventor of Regents of the University of Michigan-owned technologies that are licensed to Zydus-Cadila, to whom he is an unpaid consultant, or Alibion AG, where he holds a $2.5 \%$ equity option, and to whom he is a paid consultant. All other authors have no competing interests to declare.

\section{Additional information}

Supplementary Information The online version contains supplementary material available at https://doi. org/10.1038/s41598-021-82195-3.

Correspondence and requests for materials should be addressed to J.H.

Reprints and permissions information is available at www.nature.com/reprints.

Publisher's note Springer Nature remains neutral with regard to jurisdictional claims in published maps and institutional affiliations.

(c) (1) Open Access This article is licensed under a Creative Commons Attribution 4.0 International (c) License, which permits use, sharing, adaptation, distribution and reproduction in any medium or format, as long as you give appropriate credit to the original author(s) and the source, provide a link to the Creative Commons licence, and indicate if changes were made. The images or other third party material in this article are included in the article's Creative Commons licence, unless indicated otherwise in a credit line to the material. If material is not included in the article's Creative Commons licence and your intended use is not permitted by statutory regulation or exceeds the permitted use, you will need to obtain permission directly from the copyright holder. To view a copy of this licence, visit http://creativecommons.org/licenses/by/4.0/.

(C) The Author(s) 2021 